Western University Scholarship@Western

Digitized Theses

Digitized Special Collections

1974

\title{
On The Possibility Structure Of Physical Systems
}

William George Demopoulos

Follow this and additional works at: https://ir.lib.uwo.ca/digitizedtheses

\section{Recommended Citation}

Demopoulos, William George, "On The Possibility Structure Of Physical Systems" (1974). Digitized Theses. 765.

https://ir.lib.uwo.ca/digitizedtheses/765

This Dissertation is brought to you for free and open access by the Digitized Special Collections at Scholarship@Western. It has been accepted for inclusion in Digitized Theses by an authorized administrator of Scholarship@Western. For more information, please contact tadam@uwo.ca,

wlswadmin@uwo.ca. 
ON THE POSSIBILITY STRUCTURE - OF PHYSICAL SYSTEMS

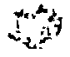<smiles>CC[18OH]</smiles>

William Demopoulos

Department of Philosoply

Program in Philosophy of Science

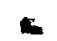

Submitted in partial fulfillment of the requirements for the degree of Doctor of Philosophy

Faculty, of Graduate stúdies The University of Western Ontario London, Ontario August, 1974 .

(c) William Demopoulos 1974. 
This dissertation is a contribution to the theory of . theories. Speciffelly, it concerns the interpretation of principle theories as opposed to constructive theories. The distinction is due to Einstein, and is basfally this: In the case of constructive, theories the idea is to reduce. a wide class of diverse systems to component systems of a " particular kind. The existence claims to which theories of this type have led are well known. (The molecular hypothesis of the kinetic theory of thermodynamic systems is an example.) Classical discussions of the reality of theoretical concepts have focussed on constructive-theories. : Principle. theories have a differtnt aim. These theories introduce abstract structural constraints which events are held to." satisfy. Theories of space-time structure provide the most - accessible illustration of principle theories. In this work the concept of a principle theory is extended to include.". theories of logical structure. j

Interpretations .of principle theories show in what fundamental respects they are related to preceding theories. For example, the special theory of relativity represents the $\therefore$ transition from Newtonian mechanics to Maxwell's electrodynamics as ịnolving a modification of the structure of space-time. It is in this sense that the special theory of relativity is an interprètation of classical electrodynamics.

Classical mechanics and quantum mechanics are represented as principle theories of logical structure. (Theories of 
this type ape called "statistical theories" or sometimes, "phase space theories".) The logical structure of a physical system is understood as imposing the most general constraint on the occurrence and non-occurrence of events.

The logical structures of quantum mechanics include the Boolean algebras of classical mechanics. Such structures represent the possibility structure of events, that is, rokghly speaking, they represent the way in which the properties of a physical system hang together. The quantum theory. has shown that significantly different assumptions may be made concerning this structure.

Chapter II formulates a general concept of completeness. applicable statistical theories. The analysis arises naturally from the cdnsideration of Gleason's theorem and its corollaries and depends on the notion of a proper. extension of a statistical theory. Extensions are defined atis

relative to a category of algebraic structures pepresenting the phase spaces of the theory and a sujtable concept of statistical state. Basically, complete statisțical theories have no-proper extensions. This notion of completeness is a mathematical propqrty of a certain class of algebraic structures rather fan a metamathematical one. There exists an important model-theoretic connection between completeness and the formal theory of this class of structures; this is expląined in Chapter I. But the concept of, completeness does not depend on this connection.

A ctonsequence of this analysis is that classical mechanics 
and quantum mechanics, are complete in exactly the same sense. In neither theory do there exist extensions in the category of algebraic structures associated with their phase spaces; As principle theoriés, classical méchanics and quán'tum mechanics specify different kinds of constraints on the possible events open to a physical.system, and each theory is complete relative to the category of algebraic structures defined.

The charactenistic feature of the logical structures of quantum mechanics is the failure of semi-simplicity, which always holds in Boolean algebras. Chapter III examines the theory of truth for phase space theories. If the disçussion" of this chapter is correct, semi-simplicity is irrelevant to the classical concept of truth as correspondence, though it is decidedly not irrelevant to the classical theory of logical structure. This means that the concept of truth is the same in both classicat and quantum mechanics; in particular, both theories are bivalẹnt. 


\section{PREFACE}

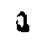

- This dissertation, consists of three papers which were prepared for'separate publication. They appear here without modification. With the exception of Chapter II, each paper is largely self-cöntained. This has led to a slight overlap in the mathematical exposition of chapters "I and III. Chapter I, "The Interpretation of Quantum Mechapics", - was written in collaboration with Jeffrey Büb. Numbers of works cited refer to the-Referendes following each chapter. The Bibliográphy is cumulative list of works cited.

Professors Leach, Hockney, and Hooker of the Philosophy of Science Program at, the University of Western Ontario provided an atmosphere in which I could freely pursue my research interests. Without their support, it is unlikely that this work would have been completed.

I wish to thank Jeffrey Bub and Hilary Putnam for introducing me to the issues dealt with in.this thesis. 
CERTIFICATE OF EXAMINATION $\ldots \ldots \ldots \ldots \ldots \ldots \ldots$ ii

ABSTRÄCT $\ldots \ldots \ldots \ldots \ldots \ldots \ldots \ldots \ldots \ldots \ldots \ldots \ldots \ldots \ldots \ldots \ldots \ldots \ldots$

PREFACE $\ldots \ldots \ldots \ldots \ldots \ldots \ldots \ldots \ldots \ldots \ldots \ldots \ldots$

TABLE OF CONTENTS, .................... vii

CHAPTER I - THE INTERPRETATION OF QUANTUM MECHANICS. I

0. Introduction $\ldots \ldots \ldots \ldots \ldots \ldots \ldots \ldots \ldots \ldots$ I

1. Space-Time Theories ................ 4

2. Phase-Space Theories $\ldots \ldots \ldots \ldots \ldots \ldots \ldots \ldots{ }_{8}$

3. Validity and Imbeddability ........... 15

4. 4. The Basic Problem .................... 31

5. Alternative Representations .......... 40 .

6. Conclusion ...................... 44

${ }^{-} \quad$ Footnotes ${ }^{*} \ldots \ldots \ldots \ldots \ldots \ldots \ldots \ldots \ldots \ldots$. 45

References ......................... 48

$\therefore \quad$ References $\ldots \ldots \ldots \ldots \ldots \ldots \ldots \ldots \ldots \ldots \cdots \cdots$

CHAPTER II - FUNDAMENTAL STATISTICAL'THEORIES ..... 51

0. Introduction $\ldots \ldots \ldots \ldots \ldots \ldots \ldots \ldots \ldots \ldots \ldots . . .61$

1. The completeness Problem for

Statistical Theories ............ 55

References ......................68

CHAPTER III - THE POSSIBILITY STRÜCTURE OF ...

PHYSICAL SYSTEMS $\ldots \ldots \ldots \ldots \ldots \ldots 70^{\circ}$

$00^{\circ}$ Introduction $\ldots \ldots \ldots \ldots \ldots \ldots \ldots \ldots \ldots \ldots \ldots$ 
1., Preliminary Notions ............... 75

2. Partial Boolean Algebras and.

Ōrthomodular Poset's

3. Applications to the Problem of Hidden

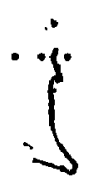

$+$ Variables ........................ 86

4. Idempotents as Propositions ............ 92

5. "Anomolies" ....................... 104

References $\ldots \ldots \ldots \ldots \ldots \ldots \ldots \ldots \ldots \ldots \ldots \ldots$

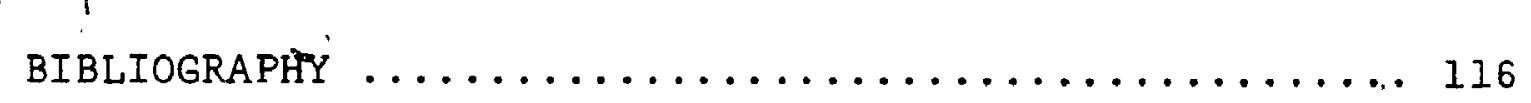

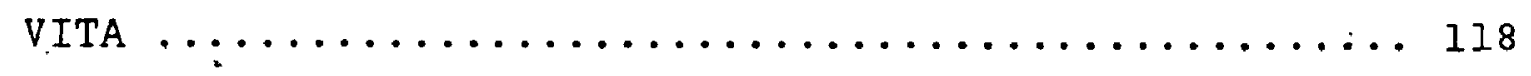

-

. 
The author of this thesis has granted The University of Western Ontario a non-exclusive license to reproduce and distribute copies of this thesis to users of Western Libraries. Copyright remains with the author.

Electronic theses and dissertations available in The University of Western Ontario's institutional repository (Scholarship@Western) are solely for the purpose of private study and research. They may not be copied or reproduced, except as permitted by copyright laws, without written authority of the copyright owner. Any commercial use or publication is strictly prohibited.

The original copyright license attesting to these terms and signed by the author of this thesis may be found in the original print version of the thesis, held by Western Libraries.

The thesis approval page signed by the examining committee may also be found in the original print version of the thesis held in Western Libraries.

Please contact Western Libraries for further information:

E-mail: libadmin@uwo.ca

Telephone: (519) 661-2111 Ext. 84796

Web site: http://www.lib.uwo.ca/ 
Chapter I

The Interpretation of Quantum Mechanics

...no solution of the problem is. possible as long as in adherence to the tendencies of Huyghens and Mach one disregards the structure of the world.

Hermann Weyl ([21] p. 105)

0 . Introduction

The quantum theory is interpreted in the technical (semantical), sense. By an interpretation of. quantum mechanics we mean something much less precise. As a rough approximation, • an interpretation of a theory should show in what fundamental respects the theory is related to preceding theories. In the case of the quantum theory this means understanding the transi-. tion from classical mechanics to elementary (i.e. non-relativistic) quantum mechanics.

To begin"with, we distinguish between two types of physical theory: 'principle' theories and 'constructive' theories. ${ }^{1}$ The difference is basically this: In the case of constructive theories the idea is to reduce a wide class of diverse systems to component systems of a particular kind. The existence claims to which theories of this type have led are well known - especially in the case of the molecular hypothesis of the kinetic theory. The classical discussions of the reality of theoretical concepts have focussed on theories of this ctype. Principle theories have a different aim These theories introduce abstract, structural constraints which events are held to satisfy. ${ }^{2}$ 
In this paper, classical ard quantum mechanics are represented as a particular type of principle theory. We call theories of this type 'phase space theories' or 'theories of logical structure' since the type of structural constraint they introduce concerns the lígical structure of events, and this is given by the phase space of the theory. 3 'The logical structure of a physical system is understood as imposing the. most general constraint on the occurrence and non-occurrence of events.

In Section $I$ we consider the concept of an abstract

- structural" constraint in the more familiar context of spacetime theories. This motivates the preliminary discussion, in Section 2, of our concept of logical structure.

Section 3 presents an elementary, characterization of the imbeddability relations between the phase space structures of classical and quantum mechanics. We' mean this in the technical sense, i.e., in terms of the validity of elementary or first-order propositional. Formulae. The characterization depends on Kochen and Specker's crucial Theorem 4.4 As is well-known, the set $S$ of statistical states of the quantum theory does not contain any states that are dispersion free. In Section 4 we discuss this in the light of oKochen and Speckerin's Theorem I. In this section, we also compare the quantum theory with classical stafistical mechanics' We conclude this section by relating our interpretation of the quantum theory in terms of logical structure to the original proposal of Birkhoff and von Neumann [2]. 
0

3

3

The mathematical discussion of this paper is based on Kochen and. Specker's concept of a partial Boolean algebra. In Section 5 , we consider two alternative representations of Hilbert space: orthomodular partially ordered sets (posts) and orthomodular lattices'. 
1. Space-Time Theories

is.

The fol owing discussion is based on the formulations of Anderson [1] and Traytman [18,19]. However the central idea of this section, viz; the distinction between coordinate transformations and symetries, originätes with Weyl. (See, 1 e.g. [219, Section 13.)

$\circ$

c.

Denóte by $E$ the set of all possible histories of physical systems, and by $\underline{\underline{F}}$ the subset of physical histories that may actualfy"occur; i.e:, the histories in $\underline{\text { I }}$ are dynamically possible or allowed by the physical laws. The structural corstraints of a space-time" theory may be understoo"d in terms of the concept of a symmetry.' By this we mean an automorphism of $E$ on $E$ which carries $\underline{F}$ into $F$. Symmetries thus proserve the laws of motion, which determine the subset $\underline{F}$ of $\underline{E}$. The space-stime stricture of a physical theory is given by the invariants of its symmetry group.

It is clearly possible to restrict $\underline{F}$ on the asumption that a particular symmetry group obtains; we are then restricting the character of physical laws on the'basis of space-time structure For examper the transition from Newtonian mechanics to special pelativity consists in a hodification of the symmetry group from the inhomogeneous' Galilean group to the Poincar group (inhomogeneous Lorentz group). The two fundamental invariants of the classical symmetry group, the Euclidian metric, and absolute time, aro dropped altogether. Space-time structure is determined by the Maxwell-Lorentz theory, and the classical laws are modified: 'new 'law of motion for rapidy moving mass points. 
A theory of space-time structure, if correct, tells us something perfectly objective about the world. There is an important misconception of the relation of relativity principles to the role of coordinates in physics which suggests that at least the choice of space-time metric is largely, if not wholly, a matte of convefition or descriptive simplicity. We discuss this matter here since it anticipates issues which will arise later in connection with the quantum theory.

To see what is involved, it suffices to consider the principle of general relativity. To begin with, we distinguish this pringiple from the principle of general covariance. As we use the term (cf. Anderson [1], Section 4.2), generally covariant theories have the property that the transform of $a$. solution of an equation is the solution of the transformed equation for 'arbitrary' coordinate transformation's. That is to say, general coveriance requires that the coordinates should not occur essentially in the formulation of physical faws. Roughly speaking, the possibility of a generally covariant formulation of physical laws is mainly a mathematical development; which was initiated by Minkowski.

In going from special to general relativitys the symmetry group is enlarged to include all diffeomorphisms, i.e. all maps which preserve the topological and differential structure of space-time. In the general theory metrical structures do not occur among the invariants of the spacetime symmetry group. This is expressed by saying that the" 
metric is not. an absolute element of the general theory, but a dynamical variable which appears as a component in the histories in $\underline{F}$. The local validity of the special theory requires that for infinitesimal regions of space-time the metric must assume ( $\dot{\mathrm{f}} \mathrm{lat})$ Minkewskian values.

The important point to notice in the transition from special to general relativity theory is that it concerns the symmetry group, not merely the covariance group of the theory. In contrast to general relativity, the principle of general covariance is compatible with the existence of a symmetry group which is properly included in the set of all diffeomorphisms. For example, in, a generally covariant formulation of special relativity, we may replace the coordinates by their curvilinear transforms. But this leaves invariant the Minkowski tensor which represents an absolute element of the special. theory. In general relativity, there are no absolute metrical elements.

So long as these two principles are not kept clearly distinct, the generalization of a relativity principle, together with the comesponding change in space-time structure which this induces, will appear to be a purely formal development. This is because it deems plausible to view a change in the covariance group of a theory as largely a matter. of mathematical conveniehce. But leven if this were true, it would be irrelevant to the interpretation of relativity principles; since they concern the symmetry group, not the covariance group of the theory. Similarly, hypotheses concerning the metric depend on relativity principles, and thus 


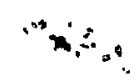

on the symmetry group, not the covariance group of the theory. But the character of the symmetry group, and therefore the metrical structure of space-time, is independent of how we describe the dynamically possible histories in $\mathrm{E}$. So even if it were possible to show that the choice of covariance group is conventional, nothing would follow concerning the choice of symmetry group.

(Notice, even the claim that the covariance group is conventional cannot be completely correct, since the requirement of general covariance restricts the class of mathematical objects which may represent physical magnitudes, and, to this extent, restriets the actual content of the theory. On this see Trautman's discussion.) 
2. Phase Space Theories ${ }^{6}$

The fundamental problem for a phase space theory is the representation problem. It is required to find a phase space structure and a probability algorithm which correctly represents the totality of all possible events associated with a certain class of physical systems. In classical particle mechanics an event is represented by a point in a subset $\Omega$ of. $6 \underline{\mathrm{N}}$ dimensional Euclidian space, where the $6 \underline{\mathrm{N}}-$ tuple $\left(q_{1}, \ldots, q_{3 N}, p_{1}, \ldots, p_{3 N}\right)$ of real numbers denotes the coordinates, of position and momentum of the $\underline{N}$ components. In quantum mechanics an event is represented by a ray in. a separable Hilbert space $H$. In this section, we confine our attention to phase space structures.

Consider the set of all intervals of the real line, $\underline{R}$, half-open (on the right). The Borel subsets of $\underline{R}$ are the sets contained in the $\sigma$-ring generated by this set. A theoretical proposition about the system asserts that the value of a physical magnitude lies in one of these intervals. In the case of basic propositions (i.e., basic theoretical propositions) the intervals are atoms in the field of Borel subsets of $\underline{R}$ :

We may imagine that the propositions of a phase space theory express the result of ideal - i.e., non-interfering - I measurements. In the case of basic propositions, the measurements are also infinitely precise.

Now consider the system at a particular instant. The greatest lower bound $\Lambda_{i}\left\{a_{i}\right\}$, $i \varepsilon I$ ( $I$ is just some index set) of the set of all the basic propositions true of the system 
at that instant is called an atom or atomic proposition. 'Each' atomic proposition determines an ultrafilter in the algebra of theoretical propositions.

Notice, theoretical propositions are algebraic objects and the structure of theoretical propositions is an algebraic structure of a certain kind. For example, in the commutative algebra $\underline{R}^{\Omega}$, theoretical propositions are associated. with the characteristic functions of the Borel subsets of $\Omega$; in the set of self-adjoint operators on a separable Hilbert space, theoretical propositions correspond to the projection operators. We adopt the following notational convention: I denotes the algebra of theoretical propositions of an arbitrary phase space theory; $\underline{C}$, the algebra of propositions of classical mechanics, and $\underline{Q}$, of quantum mechanics.

The phase space of the theory provides an alternative way of viewing this structure in terms of the topology of the space. For example, in the case, of classical mechanics, the points in $\Omega$ correspond one-to-one with máximally consistent sets of theoretical propositions, i.e., with ultrafilters in the Boolean algebra of theoretical propositions. Now let $\underline{S}(\underline{C})$ denote the Stone space of $\underline{C}$. (the set of all ultrafilters. in $\underline{c})$. The Stone isomorphism $h: \underline{C} \underline{\underline{S}}(\underline{c})$ which maps a theoretical proposition onto the set of ultrafilters which contain it preserves the structure of $\underline{C}$. Because of the correspondence between the points of $\Omega$ and the litrafilters of $\underline{S}(\underline{C})$, we may. replace the Stone space of $\underset{c}{C}$ by $\Omega$. Then $\Omega$ is the Boolean space of $\underset{C}{\mathrm{C}}$; and $\mathrm{h}$ is an isomorphism of $\underline{C}$ onto the perfect and 
reduced field $F(\Omega)$ of simultaneously open and closed subsets

of $\Omega$. Under this mapping the image of a consistent set of propositions (i.e., a proper filter in $C$ ) is a non-empty closed subset of $\Omega$. An ultrafilter in $\underline{C}$ corresponds to a singleton subset. $\{\omega\}$ of $\Omega$. The unit filter in $\underline{C}$ is associated with the whole space, and the dual of the unit filter, the zero ideal, with the empty set.

In classical mechanics, atomic events in the history of a system are represented by the points in $\Omega$, or the uItrafilters in $\underline{C}$," so that the algebra $B$ of all possible events associated with a physical system is a Boolean algebra.

The theoretical propositions of quantum mechanics form a partial Boolean algebra. This structure may be viewed as a collection $Q=\left\{\underline{Q}_{i}\right\}_{i \varepsilon I}$ of Boolean algebras such that for every $i$, $f \in I$ there is a kEI such that $Q_{i} \cap Q_{j}=Q_{k}$; and if $a_{I}, \ldots, a_{n}$ are elements of $Q=v_{i \varepsilon I}\left\{Q_{i}\right\}$ such that any two of them lie in a common $g_{i}$, then there is a $k \varepsilon I$ such that $a_{1}, \ldots$, . $a_{n} \varepsilon_{k} \cdot Q$ is a partial Boolean algebra if we restrict the aigebraic operations to elements in $Q$ which lie in a common Boolean aldebra $Q_{i}$. For the quantum theory, $Q$ is taken to be isomorphic to partial Booleàn algebra of linear subspaces. of a suitable Hilbert space, A partial Boolean algebra may be pictured as 'built up' from its maximal Boolean subalgebras. In terms of this representation, the phase space of the quantum theory is just the isomorphic collextion of Boolean. spaces corresponding to the Boolean algebras $S_{i}$. Just as in classical mechanics, an atomic event is represented by an 
ultrafilter in $Q$ or the image of this. filţer in the collection of Boolean spaces corresponding to $Q$, so that the algebra, $A$ of all possible events is a particular type' of partial Boolean algebra.

For our purposes, it is sufficiept to distinguish between Boolean and non-Boolean systems of events. The logical structure of an 'individual event is Boolean or non-Boolean according". "to. whether the physical system to which it belongs is or is not Boolean. The distinction depents on a reflexive and symme tric binary relation of compatibitity. Let $\hat{A}$ denote the set of all possible atomic events which a phase space theory associates with a physical system. If the relation of compati " bility is transitive in $\hat{A}$, the system is Boolean. This is the case in classical mechanics. The quantum theory generalizes the logical structures of classical physics by introducing, a relation. of compatibility which is not transitive in $\hat{A}$. This heads to a class of event structures which differ strongly from classical logica structures in the sense that they are not even imbeddable into a Boolean algebra.

Thio distinction between classical and non-classical - logical structures does not coincide with the distinction between classical and non-classical flmalsogics. That is, non-imbeddability into a Boolean algebra is a necessary condition for the logical structure of a system of events to be considered non-classical. But the non-elassical logics usually considered in the literature determine classical logical structures.' Each formal logic is associated with a characteristic algebra: the Lindenbaum-Tarski algebra of the logic. 
This coincides: with the logical structure determined by the formal logic. "The Pseudo-Boolean algebras ${ }^{6}$ associated with Intuitionist logic and the modal systems ố Lewis coincide with distributive lattices. So the theorem of MacNiele [14] applies: for each such algebra, .there is a Boolean imbedding". And by a result of Peremans [15]; the imbedding is constructive. The fact that there exist strongly different theoretical conceptions of the logical structure of a physical system indicates that this is as objective a component of the world as the events themselves. At least this is the major consideration in favor of realism elsewhere in science. . If it is maintained that logical sṭucture is conventional it must be possible to show that there is something which the question of logical structure does not share with other theoretical issues which would justify such an interpretation. For example, it is generally required that conventions be dispensible. Hence, if the chbice of non-Boolean logical structure were conventional, it should be possible to reformulate the theory without this choice. But the logical " structure of the quantum thepry does not have this character. Notice, in this connection, that we maintain a sharp distinction between logical structure in the sense of the phase space structure and the syntax and semantics of the formal language $L$ in which the propositions of $T$ are reconstructed. The choice of phase space is directly related to the representation problem, and therefore, to the quantum theory. The syntax and semantics of $L$ raises a completely 
different set of problems. The events hich.the propositions of $\underline{T}$ describe are represented in a certain algebraic structure.'. This is given by the phase space which the theory-associates with the.physical system considered. Now on any reconstruction of the theory, this structure is retained, whateyer the choice of $L$. Thus the syntax and semantics of $L$ - i.e., logical structure in the conventiohal sense - is not theoreticaily important.

There is another respect in, which our approach differs from accounts. which use the concept of $\dot{a}$ theoretical proposi- tion: Usually the concept of a theoretical proposition is - introduced in order to identify a phase space theory. with the pair $(\underline{T}, \underline{S})$ consisting of the system $\underline{T}$ of $\dot{i t s}$ theoretical propositions, and set $\underline{S}$ of its statistical states. Now the properties asserted by theoretical propositions are elementary or first-order properties) so they are exprossible in an elementary language. But most" theoretically interesting properties are not even general first-order properties; i.e., operties $\underline{P}$ such that a structure has $\underline{P}$ if and only. if it is a model of some (possibly infinite) set $\Sigma$ of first order sentences. For example, the property of being a Euclidean space is not a general first brder property. "This is true of other space-time properties as.well. Since, in our interpretation, such properties are an essential component of principle theories, the identification of a theory with a first-order reconstruction of the system of its theoretigal propositions is not justified. 
Finally, $i, t$ is necessary to consider the objection that the concept of logical structure introduced here involves an unjustifiable extension of 'logic'. Insofar as this is not a completely verbal issue, it overlooks several important considerations.

(A) It is possible to characterize the difference between the classical and quantum mechanical phase spaces in terms of the validity and refutability of classical tautologies, the so-called 'propositions of logic'. The concept of validity employed in this characterization is a generalization of the classical concept of validity in a straight-forward sense. Both of these points will be explained in detail in the discussion of Kochen and Specker's work.

(B) The phase space structures with which we are concerned are Boolean algebras or generalizations of Boolean algebras. From a mathematical point of view, classical propositional logic is essentially a Boolean algebra when equivalent sentences are 'identified'. There is also the well-known equivalence of the representation theory of Boolean algebras with the metatheory of classical logic.

(C) The identification of logic, or logical.structure with the syntax and semantics of formal languages is by no. means a necessary delimitation of the subject but is due to a particular point of view: viz., Formalism. Thus, in part, this paper. may be viewed as a rejection of Formalism as an adequate theory of the application of logic in physics. 


\section{Validity and Imbeddability}

This section is essentially an exposition and clarification of the work of Kochen and Specker. For the most part, we adopt their notation and terminology.

A partial algebra over a field $\underline{K}$ is a set $\underline{A}$ with a reflexive and, symmetric binary relation $\rightarrow$ (termed 'compatibility'), closed under the operations of addition and multiplication, which are defined only from $\leftrightarrow$ to $\underline{A}$, and the operation of scalar multiplication from $\underline{K} \times \underline{A}$ to $\underline{A}$. That is:

(i) $\rightarrow \subseteq \underline{A} \times \underline{A}$

(ii) every element of $\underline{A}$ is compatible,with itself

(iii) if $a$ is compatible with $b$, then $b$ is compatible with $a$, for all $a, b \in \underline{A}$

(iv) if any $a, b, c \varepsilon \underline{A}$ are mutually compatible; then . $(a+b) \leftrightarrow c, a b \leftrightarrow c$, and $\lambda a+b$ for all $\lambda \varepsilon \underline{K}$.

In addition, there is a unit element 1 whith is compatible with everry element of $\underline{A}$, and if $a, b, c$ are. mutually compatible, then the values of the polynomials in $a, b, c$ form a commutative algebra over the field $\underline{K}$.

- A partial algebra over the field $\underline{z}_{2}$ of two elements is termed a plartial Boolean algebra. The Böolean operationsmas $\checkmark$ and ' may be defined in terms of the ring operations in the, . usual way:

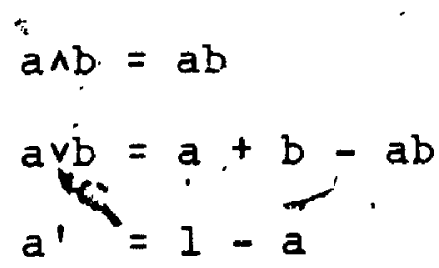

If $a, b, c$ are mutually compatible, then the values of the polynomials in $a, b, c$ form a Boolean algebra. 
Clearly, if $\underline{B}$ is a set of mutually compatible elements in a partial algebra A, then B generates a commutative sub-algebra in $A$; and in the case of a partial Boolean algebra $A, \underline{B}$ generates a.Boolean sub-algebra in $A$. Just as the set of idempotent elements of a commutative algebra forms Boolean algebra, so the set of idempotents of a partial algebra forms a partial Boolean algebra. A partial Boolean algebra may also be defined directiy in terms of the Boolean operations $\wedge, v$ and '. A pal Boofean algébra associated with a Hilbert space may be regarded as a partially ordered set with a reflexive and symmetric relation of compatibility, such that each maximal compatible subset is a Boolean algebra. We restrict the discussion now to partial Böolean algebrias. Á homomorphism, 'h, between two partial Boolean algebras, $A$ and $A '$, , is a map $h: A+A$ ' which preserves the algebraic operations; i.e. for all compatible a, beA:.

$$
\begin{aligned}
& h(a) \leftrightarrow h(b) \\
& h(a+b)=h(a)+h(b) \\
& h(a b)=h(a) h(b) \\
& h(I)=I .
\end{aligned}
$$

A homomorphism is an imbedding if it is one-to-one, and into. 'A weak imbedding is a homomorphism which is an imbedding on Boolean sub-algebras of A. More precisely, a homomorphism, $h$, of $A$ into $A^{\prime}$ is a weak imbedding if $h(a), \neq h(b)$ whenever $a \leftrightarrow b$, and $\mathrm{a} \neq \mathrm{b}$ in $A$.

A necessary and sufficient, condition for the imbeddapility of a partial Boolean algebra A into a Boolean algebra B', 
7 is that for évery pair of distinct elements a, $D E A$ there exists a homomorphism $h x \rightarrow \underline{z}_{2}$ which separates them in $\underline{z}_{2}$, i.e, such that $h(a) \neq h(b)$ in $Z_{-2}$. This is Kochen and Specker's Theorem 0 . The result depends on the semi- simplicity property of Boolean algebras, i.e. essentially, the homomorphism or ultrafilter theorem.

The counterpart of Theorem 0 for, weak imbeddability is, the fôlowing: A necessary and sufficient condition for the weak imbeddability of a partial Boolean algebra $A$ into a, Boolean algebra $B$ is that for every non-zero element $a \in A$ there exists a homomorphism, $h: \dot{A} \rightarrow \underline{Z}_{2}$ such that $h(a) \neq 0$.

A propositiona1, on Boolean function $\phi\left(x_{1}, \ldots, x_{n}\right)$ may: be regarded as a polynomial ove $\underline{z}_{2}$. To say that a particular 'propositional function,é.g. the function

$$
x_{1} \wedge\left(x_{2} \wedge x_{3}^{\prime}\right): \equiv\left(x_{1} \wedge x_{2}^{\prime}\right) \wedge x_{3}
$$

is a classical tautology, is to say that every substitution of elements from a Boolean algebra $B$ for the variables $x_{1}, x_{2}, x_{3}$ yields the unit element in $B$. We seek a generalization of this classical notion of validity to include substitutions from partial Boolean álgebras. Kocheo and Specker propgse that a propositional. function such as the above is valid in a partial Boolean algebra $A$ if every 'meaningful' substitution of elements from yields the unit element. in $A$. A 'meaningful' substitution is one which satisfies the compatibility relations; otherwise the partial operations are undefined in $A$. In this particular case, we require that the lements $a_{1}, a_{2}, a_{3}$ of $A$ substituted for. $x_{1}, x_{2}, x_{3}$ satisfy the 
conditions:

$$
\begin{aligned}
& a_{2} \leftrightarrow a_{3} \\
& a_{1} \leftrightarrow a_{2} \\
& a_{1} \leftrightarrow a_{2} \wedge a_{3} \\
& a_{1} \wedge a_{2} \leftrightarrow a_{8} \\
& a_{1} \wedge\left(a_{2} \wedge a_{3}\right) \leftrightarrow\left(a_{1} \wedge a_{2}\right) \wedge a_{3} .
\end{aligned}
$$

This notion is formalized in the following definition:

Let $a=\left\langle a_{1}, \ldots, a_{n}\right\rangle$ be an element in $A^{n}$, the $n$-fold, - Cartesian product $A^{3} \times \ldots \times A$ of the partial Boolean algebra $A$. The domain, $\underline{D}_{\phi}$, in $A$ of a propositional function $\phi\left(x_{1}, \ldots, x_{n}\right)$ is defined recursively, together with a recursive definition. of a map $\phi^{*}$ (corresponding to $\phi$ ) from $D_{\phi}$ into $A$, as follows:

(1) If $\phi$ is the polynomial 1 , then $\underline{D}_{\phi}=A^{n}$ and $\phi^{*}(a)=1$.

(2) If $\phi$ is the polynomial $x_{i}(i=1, \ldots, n)$, then $\underline{D}_{\phi}=A^{n}$, and $\phi^{*}(a)=a_{i}$.

(3) If $\phi_{0}=\psi \theta_{x}$ (where is either + or.), then $\underline{D}_{\phi}$ consists of those sequences a which belong to the intersection of the domains of $\psi$ and $x\left(i . e . a \varepsilon D_{-}{ }^{n D}-x\right)$, and also satisfy the compatibility condition $\psi^{*}(a) \leftrightarrow$ $\leftrightarrow \chi^{*}(a)$. The map $\phi^{*}(a)$ is defined by $\phi^{*}(a)=\psi^{*}(a)$ $8 x^{*}(a)$ :

The definition of the domain of a propositional function in a given partial Boolean algebra $A$ serves to make precise the notion of a 'meaningful' substitution, while the map $\phi^{*}$ $+$ defines the value of the polynomial in $A$ for edch such sustitution. 
The statement that the identity

$\phi\left(x_{1}, \ldots, x_{n}\right)=i_{1}$

rolds in $A$ is to be understood in the sense that

$$
\phi^{*}(a)=1
$$

for all aED $D_{\phi}$.

The statement that the identity

$$
\phi\left(x_{1}, \ldots, x_{n}\right)=\psi\left(x_{1}, \ldots, x_{n}\right)
$$

hidras in $A$ is to be understood in the sense that

$$
\phi^{*}(a)=\psi^{*}(a) \text {. }
$$

for all $a \varepsilon D_{-\phi} \cap D_{-\psi}$

Now, the generalized definition of validity is

this: A propositional function $\phi\left(x_{1}, \ldots, x_{n}\right)[$ i.e. a Boolean function - a polynomial, over $\underline{z}_{2}{ }^{3}$. is valid in the partial Boolean algebra, $A$ if the identity $\phi=1$ holds in $A$.

$\phi$ is refutable in $A$ if for some $a \mathbb{D}_{\phi}, \phi^{*}(a)=0$, in $A$.

$\phi$ is logically yalid in the generalized sense, i.e. Qvalid, if $\phi$ is valid in every partial Bpolean algebra A. If the choice of $A$ is restricted to Boolean algebras, this definition of validity coincides with the usual definition: the set of valid propositional formulae is just the set of classical tautologies. Thus the recursive definition of the domain of a propositional function coupled with the recursive definition of the map $\phi^{*}$ just generalizes the classical, Boolean interpretation of $\phi$.

It is important to appreciate the distinction between the validity of a Boolean function

$$
\psi \equiv x
$$

in a partial Boolean algebra $A$, and the holding of the identity 


$$
\psi=x
$$

in $A$. To say that $\psi \equiv x$ is valid in $A$ is to say that

$$
(\psi \equiv x)=1
$$

in $A$; i.e. writing $\phi=(\psi \equiv \chi)$, we require that

$$
\phi^{*}(a)=1
$$

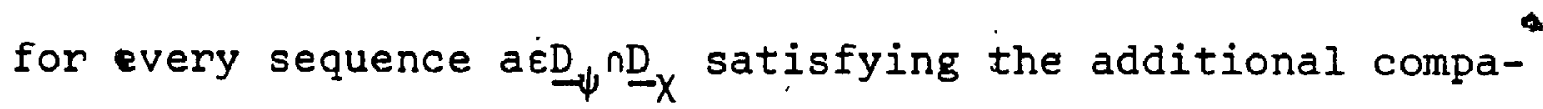
tibility condition

$$
\psi *(a) \leftrightarrow \cdot x^{*}(a) .
$$

But for the identity $\psi \equiv x$ to hold in $A$, we require that $\psi^{*}(a)=$ $x^{*}$ (a) for every sequence $a \in D_{\psi} \cap D_{-}$, not only those sequences satisfying the additional compatibility condition $\psi^{*}(a) \leftrightarrow \chi^{*}(a)$. Thus, the set of admissable sequences $a \varepsilon A^{n}$ is smaller in the case of the validity of the biconditional than in the case of the identity. If the identity holds in $A$, then certainly the biconditional is valid in $A$, but the converse is not in general true. The validity of the biconditional amounts to the holding of the identity for the restricted set of sequences which satisfy the compatibility condition $\psi^{*}(a) \leftrightarrow \chi^{*}(a)$ :

For example, let $\phi=(\psi \equiv x)$ be the classical tautology:

$$
x_{1} \wedge \cdot\left(x_{2} v x_{3}\right) \equiv\left(x_{1} \wedge x_{3}\right) \vee\left(x_{1} \wedge x_{3}\right) \text {. . }
$$

- is not only valid in every 'partial Boolejan algebra, it is also the case that the identity

$$
x_{1} \wedge\left(x_{2} \vee x_{3}\right)=\bullet\left(x_{1} \wedge x_{2}\right) \vee\left(x_{1} \wedge x_{3}\right)
$$

holds in every $A_{\text {.. }}$ For if $a=\left\langle a_{1}, a_{2}, a_{3}\right\rangle \in D_{\phi}$.

$$
\begin{aligned}
& a_{2} \leftrightarrow a_{3} \\
& a_{1} \leftrightarrow a_{2} \\
& a_{1} \leftrightarrow a_{3}
\end{aligned}
$$

But then $a_{1}, a_{2}, a_{3}$, generate a Booledn algebra. It follows 
that

and hence

$$
a_{1} \wedge\left(a_{2} v a_{3}\right)=\left(a_{1} \wedge a_{2}^{\prime}\right) \vee\left(a_{1} \wedge a_{3}\right)
$$

$$
a_{1} \wedge\left(a_{2} \vee a_{3}\right) \leftrightarrow\left(a_{1} \wedge a_{2}\right) \vee \vee\left(a_{1} \wedge a_{3}\right)
$$

Thus, every sequence a $\mathbb{E D}_{-\psi} \cap D_{\chi}$ automatically satisfies the compatibility condition $\psi^{*}(a) \leftrightarrow \chi^{*}(a)$.

In the case of a partial Boolean algebra A imbeddable into a Boolean algebra, the validity of the biconditional $\psi \equiv \chi$ in $\underline{Z}_{2}$ ( $i . e$. the classical tautologousness of the bicondi-. tional) entails the holding of the identity $\psi=x$ in $A$. Thus, in the case of imbeddability (and only in this, case):

$$
\psi \equiv x \text { is valid in } \underline{z}_{2}
$$

is equivalent to

$$
\psi=x \text {. holds in } A \text {. }
$$

This is a consequence of Kochen and Specker's Theorem 4, to which we now turn.

Kochen and Specker's Theorem 4 establishes an elementary condition for the imbeddability of a partial Boolean algebra into a. Boolean algebra. This clarifies the relationshiphetween the validity of classical tauţologies in a partial Boolean algebra $A$ and the imbeddability of $A$ into a Boolean algebra. The statement of the theorem is as follows:

(1) A necessary and sufficient condition for the imbeddability of a partial Boolean algebra $A$ into a Boolean algebra is the holding of the corresponding identity $\psi=\chi$ in A for every classical tautology

- I of the form $\psi \equiv x$. 
(2) A necessary and sufficient condition for the weak imbeddability of a partial Boolean algebra $A$ into

- a Boolean algebra is the validity in, $A$ of every classical tautology.

(3) A recessary and sufficient condition for the existence of a homomorphism from a partial

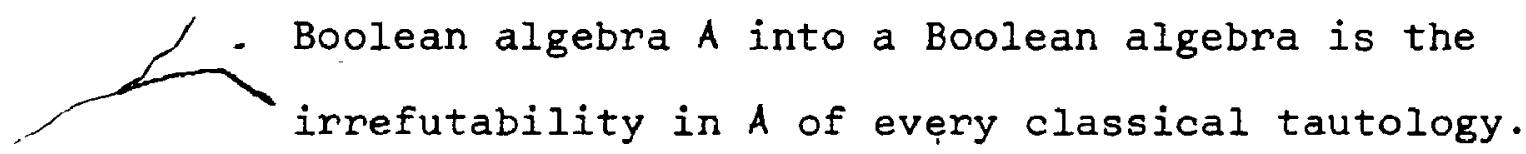

The first part of the theorem states that $A$ is imbeddable into a Boolean algebra if and only if, for every Boolean function of the form $\psi \equiv x$ which is valid in $\underline{z}_{2}$ (i.e. for which the identity $(\psi \equiv x)=1$ holds in $\left.Z_{2}\right), \psi=x$ is valid in $A$.

$\therefore$ If $A$ is imbeddable into a Boolean algebra and $\phi$ is a propositional formula not valid in $A$, i.e.

$$
\dot{\phi} *(a) \neq 1
$$

for some $a \varepsilon D_{\phi}$ in $A$, then by Theorem 0 there is a homomorphism 。 onto $\underline{Z}_{2}$ such that

$$
h(\phi *(a)) \neq h(l)
$$

i.e.

$$
\phi^{*}(h(a)) \neq 1 \text { in } \underline{z}_{2} \text {, }
$$

hence $\phi$ is not valid in $\underline{Z}_{2}$. Thus if $A$ is imbeddable into a Boolean algebra, all classical tautologies are valid in $A$.' (If we assume that the theorem holds, this may be proved directly, as follows: Suppose that for every tautology - of the form $\phi \equiv \psi, \phi=\psi$ is valid in $A$. Now let $x$ be a classical tautology. Then 
is a classical tautology, where 1 is the constant Boolean function: It follows that

$$
x=i
$$

holds in $A$, i.e. that $x$ is valid in $A_{\text {. }}$ )

The fifference between weak imbeddability and (strong) imbeddability for the set of functions valid in $A$ is just this: In the case of weak imbeddability, all the classical tautologies are valid in $A$ (and in general there are also functions valid in $A$ which are not classical tautologies). In the case of (strong) imbeddability, all the classical tautologies are valid in $A$. There may also be functions valid in $A$ which are not classical tautologies. But here we know in addition that if $\psi \equiv \chi$ is a classjeal tautology, then $\psi=x$ holds in $A$.

Thus, for weak imbeddability, if $\psi \equiv \chi$ is a classical tautology (i.e. if $\psi \equiv \chi$ is valid in $\underline{Z}_{2}$ ), we know that $\psi \equiv \chi$ is valid in $A$ (by the second part $\partial f$ the theorem), but we cannot conclude that $\psi=x$ holds in $A$. In the case of (strong) imbeddality, this inference is legitimate, i.e. from the validity of a biconditional in $\underline{Z}_{2}$, we may infer that the corresponding identity holds in $A$. This means that in the case of imbeddability we may infer theholding of the identity $\psi=\chi$

the validity of the biconditional; i.e. from

$$
(\psi \equiv x)=1 \text { in } A \text {, . }
$$

whenever $\psi \equiv \chi$ is a classical tautology, as well as the converse (which follows imediately from the definition of validity for an identity). 
Notice that we cannot conclude that only the classical tautologies are valid in $A$ if' $A$ is imbeddable into a Boolean algebra; it does not follow that if $A$ is imbeddable, and $\phi$ is valid in $A$, then $\phi$ is valid in $\underline{Z}_{2}$. For, to say that $\phi$ is valid in $A$ is. to say that

$$
\phi^{*}(a)=1 \text { in } A
$$

for every $a \varepsilon \underline{D}_{\phi}$ in $A$, and to say that $\phi$ is valid in $\underline{Z}_{2}$ is to. say that

$$
\phi^{*}(a)=1 \text { in } \underline{z}_{2}
$$

for every $a \varepsilon_{-}$in $\underline{Z}_{2}$. But the imbedding into $B$ may only use a proper subset of the sequences in $\underline{z}_{2}^{w}$ associated with the elements of $B$. We can conclude that all and only the classical tautologies are valid in $A$ only if. the imbedding is an isomorphism.

We give an exposition here only of the proof of the first part of the theorem.

The necessity of the condition is relatively easy to prove. We are required to show that the holding of the corresponding identity $\psi=x$ in $A$ for every classical tautolg $y$ of the form $\psi \equiv x$ is a necessary condition for the imbeddability of $A$ into a Boolean algebra. In other words, we are required to show that if $A$ is imbeddable, then for every biconditional $\psi \equiv \chi$ which is a classigal tautology (i.e. which is valid in $\underline{z}_{2}$ ), . the correspondipg identity $\psi=x$ holds in $A$. '.

Suppose $A$ is imbeddable into a Boolean algebra, and that $\psi \equiv x$ is a classical tautology. We must show that this entails that the identity $\psi=x$ holds in $A$, we show this by 
proving that

$$
\psi \neq x \text { in } A
$$

leads to a contradiction.

$$
\begin{aligned}
& \text { If } \psi \neq \chi \text { in } A \text {, then for some aED } \psi^{n} \underline{D}_{\chi} \text { : } \\
& \psi^{*}(a) \neq \chi^{*} \text { (a). }
\end{aligned}
$$

Now, by Theorem 0 , since $A$ is imbeddable into a Boolean algebra, for each $b, c \varepsilon A(b \neq c)$ there exists: a homomorphism $h: A \rightarrow \underline{Z}_{2}$ such that

$$
h(b) \neq h(c)
$$

and so there exists a homomorphism $h: A+\underline{Z}_{2}$ such that:

$$
h\left(\psi^{*}(a)\right) \neq h\left(\tilde{x}^{*}(a)\right)
$$

or

$$
\psi^{*}\left(h\left(a_{1}\right), \ldots, h\left(a_{n}\right)\right) \neq x^{*}\left(h\left(a_{1}\right), \ldots, h\left(a_{n}\right)\right) .
$$

In other words, $\left\langle h\left(a_{1}\right), \ldots, h\left(a_{n}\right)\right\rangle$ is an admissable sequence in $\underline{z}_{2}^{n}$ such that

$$
\psi^{*}\left(h\left(a_{1}\right), \ldots, h\left(a_{n}\right)\right) \neq x^{*}\left(h\left(a_{1}\right), \ldots, h\left(a_{n}\right)\right) .
$$

This means that $\psi \neq \chi \chi$ in $\underline{Z}_{2}$, and so the biconditional $\psi \equiv \chi$ is not valid in $\underline{Z}_{2}$, i.e. $\psi \equiv x$ is not a classical tautology, . contrary tó our original assumption.

(Notice, it would not in general be permissable to infer the non-validity of the biconditional $\psi \equiv \dot{\chi}$ from the fact that the identity $\psi=\chi$ failed to hold in a partial Boolean algebra. This inference is, however, obviously leghtimate in $\underline{Z}_{2}$ ) 。

To prove the sufficiency of the condition, we must, show that the holding of the corresponding identity $\psi=x$ in $A$ - for every classical tautology entails the imbeddability of $A$. 
into a Boolean Algebra. Kochen and Specker prove the contrapositive; if $A$ is not imbeddable into a Boolean algebra, then there exists a classical tautology $\psi \equiv \chi$ such that for some $a \in D_{-} \cap \underline{D}_{-\psi}, \psi=x$ is not valid in $A$.

Let $K_{1}$ denote the set of positive statements from the diagram of $A$, i.e. the sentences formulated in some firstorder language $L$ which describe all equations of the form $\alpha+\beta=\gamma$ or $\xi \eta=\zeta$ which subsist among elements of $\dot{A}$.

Let $\underline{K}_{2}$ be the set of sentences formulated in $L$ describing the class of Boolean algebras:

$$
\text { Write } \underline{\underline{K}}=\underline{\underline{K}}_{1} \cup \underline{K}_{2} \text {. }
$$

$\theta$

It is very important to bear in mind throughout the following that $\underline{K}$ is a subset of the sentences in $L$, the firstorder language in which the Boolean axioms are formulated, and in which relations of the form: $\alpha+\beta=\gamma$ and $\xi \eta=\zeta$ which subsist among elements of $A$ are formulated.

Now, the models of the set of sentences $\underline{K}_{I}$ are all homomorphic images of A. Hence, the cláss of all models of $\underline{K}$ cómprises all homomorphic images of A which are Boolean algebras.

If $A$ is not imbeddable into a Boolean algebra, then, by Theorem 0 , there exists a pair of elements $a, b \in A$ such that no homomorphism onto $\mathrm{Z}_{2}$ will separate them. That is, $a$ and $b$ are two distinct elements in $A$ which are identified by every. homomorphism onto $\underline{Z}_{2}$ :

If $a$ and $b$ are not separford by any homomorphism onto $\underline{z}_{2}$, then they cannot be separated by. a homomorphism into any 
Boolean algebra (by the homomorphism theorem, or the semisimplicity property of Boolean algebras). That is to say, $a$ and $b$ are identified in every model of $K$ (since the models of $\underline{K}$ äre just a class of Boolean algebras, viz. those, which are homomorphic images of $A$ ).

Thus:

$$
\underline{k} F_{h(A)^{a}=b}
$$

(where $h(A)$ is the class of Boolean algebras which are homomorphic images of $A$ ). Note that $a=b$ is to be understood here as a sentence in the first-order language $L$.

By the completeness of $L$, we have:

$\underline{K} F \mathrm{a}=\mathrm{b}$.

By the (syntactic) compactness of $L, a=b$ follows from a finite

$$
\begin{aligned}
& \text { subset } \frac{L}{2} \text { of } K_{1} \text {, where }
\end{aligned}
$$

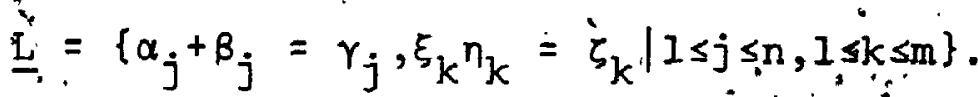

Hence:

$$
\underline{K}_{2} u \underline{L} F a=b
$$

or :

$$
\underline{k}_{2},\left\{\wedge_{j}^{\prime}, k \underline{L}\right\}+a=b
$$

where

$$
\wedge_{j, k} \underline{L}
$$

is a finite conjunction of sentences of $l$. Hence by the deduction theorem for $t$ :

$$
K_{2}+\wedge_{j} k_{k} L+a=b \text {. }
$$

Clearly, $\wedge_{j, k}=$ is logically equivalent to the conjunction

$$
\wedge_{j, k}\left\{\alpha_{j}+\beta_{j}+\gamma_{j}=0, " \xi_{k} \eta_{k}+\zeta_{k}=0 \mid 1 \leq j \leq n, I \leq k \leq m\right\}
$$

of sentences of $L$, which is logicaily equivalent to the sentence: 


$$
v_{j, k}\left\{\alpha_{j}+\beta_{j}+\gamma_{j}, \xi_{k} \eta_{k}+\zeta_{k} \mid 1 \leq j \leq n, 1 \leq k \leq m\right\}=0
$$

where the sign $v$ is to be understood as the supremum or least upper bound in the partial Boolean algebra $A$. That is,

$$
v_{j, k}\left\{\alpha_{j}+\beta_{j}+\gamma_{j}, \quad \xi_{k} \eta_{k}+\zeta_{k} \mid I \leq j \leq n, 1 \leq k \leq m\right\}
$$

denotes an element in $A$ : the least upper bound of all the elements of the form $\alpha_{j}+\beta_{j}+\gamma_{j}$, and $\xi_{k} \eta_{k}+\zeta_{k}$. The sentence asserting that, the least upper bound of all these elements is zero is equivalent to the conjunction of all the sentences asserting separately that $\alpha_{j}+\beta_{j}+\gamma_{j}=0(1 \leq j \leq n)$ and $\xi_{k} n_{k}+\zeta_{k}=0(1 \leq k \leq m)$.

It follows immediately that:

$\underline{k}_{2} \vdash\left(v_{j, k}\left\{\alpha_{j}+\beta_{j}+r_{j}, \xi_{k}^{i} n_{k}+\zeta_{k} \mid 1 \leq j \leq n, 1 \leq k \leq m\right\}=0\right)+a=b$. Write:

$$
\begin{aligned}
& \rho\left(\alpha_{1}, \ldots, \zeta_{m}\right) \underline{l} \\
& \quad=v_{j, k}\left\{\alpha_{j}+\beta_{j}+\gamma_{j}, \xi_{k} n_{k}+\zeta_{k} \mid 1 \leq j \leq n, 1 \leq k \leq m\right\} .
\end{aligned}
$$

Then:

$\underline{\mathrm{K}}_{2}+\rho\left(\alpha_{1}, \ldots, \zeta_{\mathrm{m}}\right)=0 \rightarrow \mathrm{a}=\mathrm{b}$.

Since the constants $\alpha_{1}, \ldots, \zeta_{m}, a, b$ do not occur in $\underline{k}_{2}$, they may be replaced by variables $A_{\mathrm{I}}, \ldots, \mathrm{x}_{\mathrm{m}}, \mathrm{x}, \mathrm{y}$ to obtain:

$\underline{\mathrm{K}}_{2}+\rho\left(\mathrm{x}_{1}, \ldots, \mathrm{x}_{\mathrm{m}}\right)=0 \rightarrow \mathrm{x}=\mathrm{y}$.

We have now shown that the conditional:

$$
p\left(x_{1}, \ldots, x_{m}\right)=0 ? x=y,
$$

which is to be understood as a formula in $L$, is valid in all Boolean algebras.

Let.

$\psi$ denote $x \rightarrow \rho$

$x$ denote $y \rightarrow p$ 
i.e. $\psi$ and $x$ are Boolean functions, explicitly:

$\psi$ is the function $1-x+\rho-(1-x) \rho$

$\therefore \quad x$ is the function $1-y+\rho-(1-y) \rho$.

Since :

$\rho=0 \rightarrow x=y$

is valid in all Booleanalgebras, it follows that the identity

$$
\psi=x
$$

holds in $\underline{Z}_{2}$, i.e.

$$
\psi^{*}(a)=x^{*}(a)
$$

for every sequence $a \in D_{\psi} \cap \underline{D}_{x}$. For, suppose under some substitution for the variables $x_{1}, \ldots, x_{m}$, that $\rho^{*}(a)=0$. Then because $\rho=0 \rightarrow x=y$ is valid in $\underline{z}_{2}$, we have:

$$
\psi^{*}(\mathrm{a})=x^{*}(\mathrm{a})
$$

If, under some substitution $\rho^{*}(a)=1$, we have:

$$
\begin{aligned}
& \psi^{*}(a)=1=x^{*}(a) . \\
& \text { Since the identity } \\
& \psi=x
\end{aligned}
$$

holds in $\underline{Z}_{2}$, it follows that

$$
\psi \equiv x
$$

is valid in $\underline{Z}_{2}$, i.e. that $\psi \equiv \chi$ is a classical tautology. But $\psi=x$ does not hold in $A$. For, substituting the sequence $\left\langle\alpha_{1}, \ldots, \zeta_{m}, a, b\right\rangle$ of elements from $A$ for the variables $\left(x_{1}, \ldots\right.$, $\left.x_{m}, x, y\right)$ yields the value $1-a$ for $\psi$ and $1-b$ for $x$. That is, under this valuation for and $x$ in $A$, we have

$$
\psi^{*}=1-a
$$

and

$$
x^{*}=1-b
$$

with $a \neq b$. 
Thus, in the case of a partial Boolean algebra $A$ for which there is no Boolean imbedding, there is a Biconditional, ' $\psi \equiv \chi$, whiph is a classical tautology, and a sequence $<\alpha_{1}, \ldots, \zeta_{\mathrm{Mh}}$, $a, b>\varepsilon \underline{D}_{\psi} \cap \underline{D}_{\chi}$ under which the corresponding identity, $\psi=x$, does. not hold in A. This proves the theorem. 
The set $S$ of statistical states of quantum mechanics does not "contain states which are dispersion free." This is the property of the quantum theory which generates the problem of 'interpretation': i.e., the problem is to understand the absence of dispersion free states.

A statistical state $\psi E \underline{S}$ is a map $\psi: \underline{T} \rightarrow[0,1]$ such that $\psi(1)=1$ and $\psi\left(v_{i}\left\{a_{i}\right\}\right)=\Sigma_{i} \psi\left(a_{i}\right)$ if $\left\{a_{i}\right\}$ is a disjoint sequence. That is, a statistical state is a generalized probability assignment to the theoretical propositions of the theory which satisfies the ustual. conditions for a probability measure on each maximal compatible subset of the partial Boolean algebra I. The probability algorithm of a phase space theory is a furetion $\underline{P}$ which assigns to each magnitude $\underline{A}$ and each śtate $\psi$, a probability measure on $\underline{R} \cdot \underline{P}_{A \psi}(U)$ denotes the probability that in the state $\psi$ the value of the magnitude $\underline{A}$ lies in $\underline{U}$. -For dispersion free states, the probability assigned to each magnitude reduces to an atomic measure concentrated on the value of $\mathrm{A}$. It is not difficult to show that $\psi$ is dispersion free if and only if $\psi$ is a homomorphism of $\underline{T}$ onto $\underline{Z}_{2}$ (See e.g., Gudder [8] for à proof.)

In classical mechanics, the" algebra $B$ of events is a Boolean algebra, so there is a one-to-one correspondence between atomic events a $\underline{\hat{B}} \underline{\hat{B}}$ and two-valued homomorphisms on $B$. This property is preserved in the algebra of theoretical propositions. Thus' when $B$ is a Boolean algebra, each atomic event determines a two-vadued homomorphism, and hence, a dispersion free state, on $\underline{T}$. 
Each event in $\underline{\hat{B}}$ is represented bi-uniquely by a point $\omega \varepsilon \Omega$, since the field of subsets of $\Omega$ is perfect and reduced. Each point $\omega \varepsilon \Omega$ determines a two-valued homomorphism on $F(\Omega)$, and therefore, a dispersion free state on $\underline{C}$. Conversely, since $C$ is isomorphic to $F(\Omega), \psi \circ h^{-1}$, where $h$ is the Stone isomorphism, is a statistical state on $\Omega$.

A dispersion free state on $\Omega$ may be replaced by the

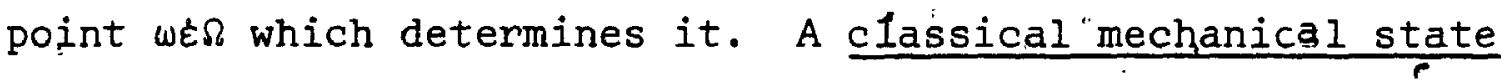
is just the phase point which determines $\psi$ when $\psi$ is dispersion free. Thus classical mechanical state corresponds to an atomic proposition in $\underline{C}$. Because $\underline{C}$, is a Boolean algebra, each atom in $\underline{C}$ determines a homomorphism onto $\underline{Z}_{2}$, and hence a dispersion free statistical state.

In a.Boolean algebra, each a $\underline{\hat{B}}$ determines a maximal propér filter $\mathbf{F}$ in $B$; similarly, in a partial Boolean algebra each aعÂ; determines a maximal proper filter $\underline{F}$ in $A$. Notice, in case $A$ is a Boolean algebra, each maximal proper filter in A may be used to define a romomorphism onto. $\underline{z}_{2}$ by the conditic, $h(a)=1$, if $a \in \underline{F}$ and $h(a)=0$ if atE. This possibility depends on the distributivity of Boolean algebras, for this impliess that maximal proper filters in $A$ are prime filters. 8 If the ultrafilters in $A$ are not prime, we may have atF and a'tE, but $I \varepsilon \underline{F}$, and therefore, ava' $\varepsilon \underline{F}$. Hence, the correspondence between 'ultrafilters in $A$ and two-valued homomorphisms on $A$ breaks down. This means that the correspondence between atomic propositions (or events) and two-valued homomorphisms breaks down: 
Notice, even if the ultrafilters in $A$ are not prime, A may be the homomorphic image of a Boolean algebra. If this is the case, there exist homomorphisms $h: A \rightarrow \underline{Z}_{2}$, however, these are not in general determined by maximal proper filters in $A$. In general, event structures which determineydispersion free states on the associated algebra of theoretical propositions include all those that may be mapped homomorphically into a Boolean algebra, since, by the homomorphism theorem, every Boolean algebra admits homomorphisms onto $\underline{Z}_{2}$. All the event structures isomorphic to or containing the partial Boolean algetra $\underline{B}\left(H_{3}\right)$ of linear subspaces of a three dimensional Hilbert space ${ }^{9}$ fall outside of this class. By, Kochen and Specker's Theorem 1, for each such A there are no homomorphiśms onto $\underline{Z}_{2}$, hence, no two-valued homomorphisms on the partial Boolean algebra $Q$ of theoretical propositions associated with A. Because of the equivalence between two-valued homomorphisms and dispersion free states, there are no dispersion free states on $Q$.

Thus the absence of dispersion frep states on $Q$ is a direct consequence of the fact that $A$ is a particular type of partial Boolean,algebra, just as the existence of dispersion free states on $\underline{C}$ is a consequence of fact that $B$ is a Boolean algebra. 10

The fact that there are no homomorphisms $\mathrm{h}: \mathrm{Q} \rightarrow \underline{Z}_{2}$ must be sharply distinguished from the question of the bivalence of the language $L$ in which the theoretical propositions, are formulated. It is trivially possiple to make bivalent as- 
- signmerits of truth values to the propositions of $Q$, and therefore, to the corresponding sentences of $L$ : Let every proposition associated with an event in an ultrafiltep in $A \cdot$ be true, and every proposition associated with an event outside the filter, false. Since the ultrafilters in $A$ are not prime filters, the bivalent assignment of truth values to $L$ is not induced by a homomorphism of $A$.onto $\underline{Z}_{2}$. But since the homomorphism theorem is equivalent to Stone's representation theorem, this is to be expected, if $A$ is strongly nori-Boolean.

In the view advanced here, events $b_{i} \varepsilon A$ incompatible

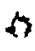
with an event $a \in A$ are excluded by the logical structure of the system; the chief advantage of the bivalent truth value assignment defined above is that it makes this fact explicit. This definition has the consequence that it is a sufficient but not a necessary condition for the truth of a disjunction that 'one of the disjuncts be true. For example, the propositional formula

$$
x_{i}+x_{j}+x_{k}-x_{i} x_{j} x_{k}
$$

in $L$ is true, whenever it is interpreted over three mutually compatible events $a_{i}, a_{j}, a_{k} \varepsilon \hat{A}$ since.

$$
a_{i} \vee a_{j} \vee a_{k}=1 \text {. }
$$

Now the trivial event (i.e., 1 ) is compatible with every event' and is a member of every filter. But, it does not follow that exactly one of every triple of mutually compatible atomic propositions is true. 
It mày be objected that such a truth vaIue assignment. is 'unintuitive'. But this is surely a psêudo-problem. For if the quantum theory is assumed, the models $M$ of $L$ are isomorphic to $\underline{B}\left(H_{3}\right)$. Therefore the ultrafilters in $\underline{M}$ are not prime filters. Thus, so far as the event structures are concerned; this property is preserved in any reconstruction of the theory. The objection is interesting only if it is coupled with a classical solution to the representation problem. But this problem is left untouched by the choice of $L$.

In the remainder of this section we compare the quantum theory with classical statistical mechanics, since for this theory it is also true that dispersion free states are not theoretically fundamental. This is done in two stages. We begin by considering why dispersion free states are not theoretically important in statistical mechanics. Mext, we examine the sense in which the description of statistical mechanics is incomple,te relativet thalewtonian description. We conclude this section with some remarks on the interpretation of Birkhoff and von Neumann:

Classical mechanics and člassical statisticàl mechanics share the same phase space as well'as'the same dynamics. Thus for statistical mechanios a dispersion free state is determined by the classical mechanical state of the system. Just as in classical mechanics, physical magnitudes $\underline{A} \varepsilon^{O}$ are associated with functions in $\underline{R}^{\Omega}=\left\{f_{A}: \Omega+\underline{R}\right\}$ from $\Omega$ into the Borel subsets of R. Each $\underline{A}$ is associated with a family of subsets of $\Omega$ by the inverse image

$$
f_{A}^{-1}(U)=\left\{w \mid f_{A}(w) \in U\right\}
$$


of the Borel subsets of $\underline{R}$ under the map corresponding to $\underline{A}$. In statistical mechanics $\Omega$ is also a sample space; so that a point $\omega \varepsilon \Omega$ is also interpreted as the phase point of a sample system in an ensemble of similar systems.

Now for a certain class $K$ of regions of the sample space, there are macroscopic magnitudes, i.e. properties of the ensemble, with values concentrated on a small subset of R. 11 "These magnitudes obey the thenomenological equations of cla cal thermodynamics. For some $A \varepsilon K$, and any distribution of phase points in $\Lambda$, the values of each macroscopic magnitude remain concentrated on a small subset of $\underline{R}$. This is to be expected; for the basic Newtonian Iaws are symmetric with respect to time, hence the law of motion of an individyal system is time-symmetric. But the phenomenological laws are irreversible; 'if the macroscopic magnitudes were not independent of the precise location of the phase point, the macroscopic laws would be reversible, not irreversible. Therefore the theoretical unimportance of the classical mechanical state is a necessary condition for the successful application of statistical mechanics to thermodynamic systems. ${ }^{12}$ The application of the laws of Newtonian physics to a thermodynamic system requires too fine a specification of the classicalmechanical state. The slightest discrepancy amplifies very rapidly and renders the initial specification theoretically useless. Because of this difficulty, wsiforgo a complete description in terms of the classical mechanical state in favor of 
an incomplete description involving a proper subset $\underline{s}^{\prime}$ of the statistical states $\psi: F(\Omega)+[0,1]$. Relative to this description, there exist magnitudes. $\underline{A}, \underline{B} \varepsilon O$ such that

$$
\underline{P}_{A \psi}(U)=\underline{P}_{B \psi}(U)
$$

for every Bore ${ }^{\prime}$ set $U \subset \underline{R}$ and every $\psi E \underline{S}$ '. $\underline{A}$ and $\underline{B}$ are equivaient with respect to the set $\underline{S}^{\prime}$ of statistical states. Therefore, $\underline{c}$ contains theoretical propositions of the form

$$
\sim f_{A}(\omega) \varepsilon U
$$

and

$$
f_{B}(\omega) \varepsilon U \text {. }
$$

which are equivalent with respeot to $\underline{S}^{\prime}$. / By extending $\underline{S}^{\prime}$ to $\underline{S}$ it is possible to distinguish these magnitudes together with the theoretical propositions corresponding to them. Thus, the statistical description in terms of $\underline{s}^{\prime}$ is incomplete relative to the classical description in the sense that the extension to $\underline{S}$ leads to an imbedding of this description into the classical description: For this reason, the absence of dispersion free states may be taken to mean that, relative to classical physics; the description of statistical mechanics is based on incomplete knowledge of the exact classical state of the system.

Now in the case of quantum mechanics, the absence of dispersion free states cannot be understood in this way. For if $A$ is a partial Boolean algebra of events corresponding to a Hilbert space of at least three dimensiotis, there is no imbedding of $A$ into a Boolean algebra; kenlee, there is no 
imbejdding of the partial Boolean algebra $Q$ of theoretical propositions associated with $A$ into a classical (i.e. Boolean) description. This follows from Kocken and Specker's Theorem 1, because the existence of "two-valued homomorphisms is a necessary condition for the imbeddability of, $Q$ into a Boolean algebra. There even exist finite subalgebras $\underline{D}$ of $Q$ which are not imbeddable into a Booleän algebra. D contains pairs of propositions such that $a \neq b$, but $h(a)=h(b)$ for every homomorphism $h: \underline{D}+\underline{Z}_{2}$. If $\underline{D}^{\prime}$ is weakly imbeddable, $a$. and $b$ will have to be incompatible. This follows immediately from the definitions of strong and, weak imbeddability, or more precisely, from Kochen and Specker's Theorem 0 and its counterpart. for weak imbeddings.

It is often suggested that the quantum theory is more vague than classical physics in the sense that there are distinctions which can be made in classical physics which are ill-defined in the quantum theory. The opposite is the case :there are finer distinctions possible in the quantum mechanical case than in the classicap case. The $a$ and $b$ above are in $a$ sense distinguishable quantum mechanically, but not classically, i.e. ${ }^{13}$ not in terms of homomorphisms anto $\underline{z}_{2}$. Intuitively, there exist completely symmetric but distinct elements in a nbn-imbeddable A.

Though Birkhoff and von Neumann recognize that the *algebra of theoretical propositions of quantum mechanics is not a Boolean.algebra, they do not consider the possibility of imbedding $Q$ into a Boolean algebra, and. thus, into a classical description. This is due to their conception of the 
role of the classical mechanical state in statistical mechanics.

In their view there are basically two reasons for ignoring the exact classical description in statistical mechanics: first, it is 'convenient' to do so, and secondly, knowledge of the phase point requires a degree of precision which it is impossible to obtain experimentally. While this is certainly true, it is not an analysis of the relationship between the two theories. Statistical mechanios, can ignore the classiçal state because it deals with irreversible pro-

s. cesses, and these must be independent of the exact phase point of the system. This account is combined with a particularly. naive confusion of reference with evidence, Birkhoff and von - Neumann are thus led to the view that it is meaningless to suppose that the system is "always in a state corresponding to a point in $\Omega$; i.e., the exact classical description is not relevant to statistical mecharics on largely independent, epistemological grounds. But blurring the distinction between the world, and our knowledge of the world, makes it impossible to distinguish a Boolean description based on incomplete knowledge from a complete non-Boolean description. Since the concept of the exact state of a system is considered funda-. mentally incoherent, the non-Boolean character of $Q$ may merely express the impossibility of knowing the complete classical state. Because of this unclarity, Birkhoff and von Neumanr. have only succeeded in reformulating the orthodox interpretation. ${ }^{14}$ Though their discussion is couched in țerms of the logical structure of quantup propositions it suffers from all the ambiguities of the conventional view. 
5. Alternative Representations

In this section we consider alternative representations of the Hilbert space structure of quantum mechanics, viz.: orthomodular posets, and orthomodular latticos. ${ }^{15}$ It is clear that the partial Boolean algebra of subspaces of a Hiibert space may be extended to an orthomodular poset by simply defining the order relation in each maximal Boolean sub-algebra in the usual way; and even to an orthomodular lattice by defining g.l.b. and l.u.b. for incompatible elements. Thus the mathematical differences are not essential. In the lattice and poset approaches there are basically two.properties that are held to distinguish $Q$ from $\underline{C}$ : nondistributivity, and the existence of incompatible pairs.Neither corresponds to non-imbeddability. Because of this, interpretations based on theserpepresentations suffer from the same ambiguity as the view of Birkhoff and von Neumann. The orthomodular lattice ${ }^{16} \mathrm{H}_{2}$ of Iinear subspaces of a two dimensional Hilbert space is isomorphic to the lattice of subspaces through a point in ordinary two-dimensional Euclidian space, In this pepresentation, compatibility corresponds to orthogonality, i.e. two Iinear subspaces are compatible if and only if they are orthogonal in the sense of elementary geometry., (Thus $a \rightarrow b$, if $a$ is a subsace of $b \%$ ). Joins, meets, and complements correspond to spans, intersectionis, and orthocomplements. The unit of the lattice is the whole space, and; the zaro is associated with the zero-dimensional subspace. or origin. It is obvious from Kochen and Specker's Theorem 0 
that the partial Boolean algebra $\underline{B}\left(H_{2}\right)$ associated with $\mathrm{H}_{2}$ is imbeddable into a Boolean algebra.

Since $\mathrm{B}\left(\mathrm{H}_{2}\right)$ is imbeddable into a Boolean algebra, the -gistributive law

$a \wedge(b \vee c) \equiv(a \wedge b) \vee(a \wedge c)$

of classical logic holds. In fact the identity holds in $\underline{B}\left(\mathrm{H}_{2}\right)$. This is not peculiar to $\mathrm{B}\left(\mathrm{H}_{2}\right)$, for the distributive identity. is $Q$-valued, i.e. valid in all partial Boolean algebras. ${ }^{17}$ $\checkmark$ Clearly, non-distributivity, in the sense of failure of the distributive law of classical logic, depends on the definition of validity. More interestingly: even when the propositional (i.e. Boolean) functions in $L$ are interpreted as lattice polynomials, the failure of the distributive law does not correspond to non-imbedảabìitity.

Similarly, there are clearly incompatible elements in $\mathrm{H}_{2}$; yet $\mathrm{H}_{2}$ may be imbedded into a Boolean algebra. So the existence of incompatible pairs must be distinguished from non-imbeddability.

The work of fierler and Schlesinger [22] shows that there always exists a map $h: A \rightarrow B$ from an orthomodular poset $A$ into a Boolean algebra which preserves the ordering and orthocomplementation. That is,

(i) if $a \leq b$, then $h(a) \leq h(b)$

(ii) $h\left(a^{\prime}\right)=h(a)^{\prime}$.

The map is also monomorphic so that. (iii) if $h(a) \leq h(b)$, then $a s b$; - Notice it does not follow that such a map preserves lattice meets and joins, for although the ordering of elements 
above and below $a$ and $b$ is preserved in the image $h(A)$ of $A$ in $B$, there may be an element of $B$ smallen (in B) than the a image of any element above $\mathrm{a}$ and $\mathrm{b}$ in $\mathrm{A}$, so that this element would qualify as $h(a) \vee h(b)$ and not $h(a \vee b)$. That is, for any $x \in A$, such that $a v b \leq x$ in $A$,

$$
h(a v b) \leq h(x),
$$

but the smallest element above $h(a)$ and $h(b)$ might be an element in $B$ which is not the image of any element in $A$. Zierler and Schlesinger show further that there does not in general exist a map satisfying conditions $(j)-(i i i)$ which also preserves the lattice operations for compatible elements. Now this is lready clear from the work of Kochen and Specker, since independently of the question of the preservation of order, meets and joins cannot be preserved in the case of partial Boolean algebras associated with Hilbert spaces of three or more dimensions: On, any representation, what is fundamental about the non-Boolean structures of quantum mechanics is that they are not imbeddable into a Boolean algebra, and this depends on the fact that $\leftrightarrow$ is not tgansitive in $\underline{\hat{A}}$. In this sense, the order structure is redundant.

(Notice non-transitivity of $\leftrightarrow$ in $\underline{\hat{A}}$ is not the same as non-transitivity iof $\leftrightarrow$ in $A$. For every element in $A$ is compatible with the unit. Thus if compatibility is transitive in $A$, then every element is compatible with every otler $r a \leftrightarrow 1$ and $1 \leftrightarrow b$ implies $a \leftrightarrow b$. That is, there are no incompatible 'pairs. Conversely if $\leftrightarrow=\underline{A} \times \underline{A}, \leftrightarrow$ is obviously transitive. 
43

Thus transitivity of $\leftrightarrow$ in $A$ is equivalent to $\leftrightarrow=\underline{A} \times \underline{A}$.)

One final point: It seems natural to understand the generalization of the order relation in a Boolean algebra as a generalized implication. However this leads to difficulties. By a result, of Fay [6], implication cannot be defined as in classical logic by

$$
a \Rightarrow b \text { if and only if } a^{\prime} v b=1 \text {. }
$$

For in an orthomodular pose or orthomodular lattice, if the relation

$$
a^{\prime} \vee b=1
$$

is transitive, the pose or lattice is a Boolean algebra.

For this reason it has been argued (e.g. by Gadder and Greechie [9]) that the transition from classical to quantum mechanics is not properly concerned with logic. But in view of Kochen and Speaker's Theorem 4, this is obviously a purely verbal issue. 
6. Conclusion

It remains to be shown how the discussion of this paper leads to a solution of the 'measurement problem'. This subject, together with a complete discussion of the role of probabilities on non-Boolean event structures, will be dealt with in a separate paper. The present paper clarifies what is required of an interpretation of the quantum theory: The problem is to explain the transition from classical mechanics to quantum mechanics, given. that the set $\underline{s}$ of statistical states of the quantum theory does not contain dispersion free states. It also explains the sense in which quantum mechanics and classical mechanics theories of the world's logical structure. This, in conjunction with Theorems 1 and 4 of Kochen and Specker, completely solves the problem of interpretation. Clarification of the problem of hidden variables, in the sense of the importance of Gleason's Theorem and its corollaries, is immediate: such results have the character of completeness theorems for the logical structures of quantum meahanics. The whole discussion rests on the distinction. between logical structure in the sense of the syntax and semantics of a formal language, and the logical structure of events. This distinction is completely analgous to the one drawn in Section 1 between coordinate transformations and symmetries. Only the first component of each pair involves. conventional elements. Logical structure and space-time symmetries are objective structural properties of the world. 
1. This distinction is suggested by Einstein [4]. We have retained his terminoldgy. Cf. also [5], pp. $53 f f$.

2. There are theories which are clearly both constructive and principle theories, e.g. classical statistical mechanics.

3. Notice that in Bub [3], 'phase space theory' denotes a classical phase space theory. We extend the use of the term here to include any theory in which the concept of logical structure occurs explicitly. .

4. This theorem is proved in [13]. Unless otherwise ind cated, all references to Kochen and Specker, are to this paper.

5. This paper assumes some acquaintance with the representation theory of Boolean algebras. See, e.g. Sikorski [17], Chapter I.

6. For a chracterization of these algebras, see Rasiowa and Sikorski [16].

7. Kochen and Specker the term'commeasurability' to refer: to this relation, clearly suggesting, that the relation should be understood in terms of simultaneous measurability. This is at least milsleading, since the simultaneous measurability of two magnitudes is a consequence of the fact that they are compatible; but compatibility is not operationally definable in terms of simultaneous measurability. [Cf. the discussion below (i.e. Section 4 ) of Birkhoff and von Neumann.] 
8. See Rasiowa and Sikorskị [16], Chapter I, Section 9, for a discussion of this point.

9. For definiteness, we restrict the discussion of this section to partial Boolean algebras of this class.

10. In general, for a Hilbert space of three or more dimensions, all possible statistical states on the partial Boólean algebra of.linear subspages are generated by the statistical operators according to the algorithm of the quantum. theory. That is to say, the probability algorithm of the theory generates all possible statistical states on $Q$. (This is essentially the content of. Gleason's theorem [7].) Yet the set. $\underline{S}$ of statistical states does not contain states "o which afe dispersion free. So, by the equivalence,between two-valued homomorphisms ànd dispersion free states, an extension of the theory which recovers the fcorrespondence between events and two-valued homomorphisms does. not exist.

11. $H$ is the class of Borel subsets of $\Omega$ modulo Borel sets of Lebesgue measure zero. Thi's class is identical with $\therefore$ the class of Lebesgue measurable subsets of $\Omega$ modulo sets of Lebesgue measure zero (see e.g. Halmos [10]; Section 15).

12. This has the character of a randomness assumption. It is also a sufficient condition for applying statistical mechanics, to thermodynamic systems. For a thorough: discussion see var Kampen [20], Chapter 1.

13. By Stone's epresentation theorem, every pair of distinct elements in a Boolean algebra must "be distinguishable 'by a homomorphism onto $\underline{z}_{2}$. 
14. In its original form; Heisenberg's interpretation was compatible with the existence of a classical mechanical state. This assumption was later rejected by Heisenperg and Bohr and replaced by the thesis that an atomic system cannot be significantly described independently of a measurement process.

15. Birkhoff and von Neumann assume an orthocomplemented, modular lattice. This assumes more structure than an orthomodular lattice. (See Jauch [11], Chapter 5, Section 6 , for a more detailed discussion of this point.) For our purposes, the difference is not important, and everything said concerning orthomodular lattices may be extended to the lattice of Birkhoff and von Neumann.

16. For simplicity of exposition we restrict the discussion. to lattices.

17. Cf. Section 3, above. For a generalization, se Kochen and specker $[12]$, Section 6 . 
[1] J. L. Anderson, Principleds of Relativity Physics, Academic Press, 1967.

- [2] G. Birkhoff and J. Von Neumann, "The Logic of Quantum Mechanics", Annals of Mathematics 37 (1936) 823-843.

[3] J. Bub, "On the Possibility of a Phase Space Reconstruction of the Quantum Statistics: A Refutation of the Bell-Wigner Locality Argument", Foungations of Physics $\underline{3}$ (1973) $29-44$.

[4] A. Einstein, "What is the Theory of Relativity?", (1919) Essays in Science, Philosoppical Library, 1934.

[5] A. Einstein, Autobiographicel Notes (1949) Albert Einstein: Philosopher-Scientist (ed. by P.A. Schilpp), Harper, 1959.

[6] G: Fây, "Transitivity of Implication in Orthomodular Lattices", Acta Sci. Math. (Szeged) 28 (1967) 267-270.

[7] A. Gleason, "Measures on Closed Subspaces of Hilbert Space", Journal of Mathematics and Mechanics 6 (1957) $885-893$.

[8] S. Gudder, "On. Hidden-Variable Theories", Journal of Mathematical Physics 11 (1970) 431-436.

[9] S. .Gudder and R. Greechie, "Is Quantum Logic a Logic?". Helv. Phys. Acta 44 (1971) 238-240.

[10] P. Halmos, Lectures on Boolean Algebras, Van Nostrand, 1963.

[11] J. Jauch; Foundations of Quantum Mechanics, AddisonWesley, 1968 . 
[12] S. Kochen and E. P. Specker, "Logical Structures Arising in Quantum Theory", The Theory of Models, 1963 Symposium at Berkeley, compiled by J. Addison et.al. NorthHolland, 1965 .

[13] S. Kochen and E. P. Specker, "The Problem of-Hidden Variables in Quantum Mechanics", Journal of Mathematics And Mechanics 17 (1967) 59-87.

[14] H. M. MacNeille, "Partially Ordered Sets", Trans. Am. Math. Soc. 42 (1937) 416-460.

[15] W. Peremans, "Embedding of a Distributive Lattice into a Boolean Algebra", Nederl. Akad. Wetensch. Indag. Math. $19(1957) 73-81$.

[16] H. Rasiowa and R. Sikorski, The Mathematics of Metamathematics, P.W.N:, 1963.

[17] R. Sikorski, Boolean Algebras (2nd ed), Springer-Verlag, 1964.

[18] A. Trautman, Lectures on General Relativity, Brandeis Summer Institute, 1964,..Prentice Hall, 1965.

[19] A. Trautman, The General Theory of Relativity, Nuclear Energy Information Center of the Polish Government, 1968.

[20] N. G. van Kampen, "Fundamental Problems in the Statistical Mechanics of Irreversible Processes", Fundamental Problems in Statistical Mechanics," Proceedings of the NUFFIC International Summer Course in Science at Nijenrode Castle, The Netherlands, 1961, compiled by E.G.D. Cohen, North-Holiand, 1962. 
[21] H. Weyl, Philosophy of Mathematics and Natural Science (Rev. ed.), Princeton: Princeton University Press, 1949.

[22] N. Zierler and M. Schlessinger, "Boolean Embeddings of Ortho-Modular Sets and Quantum Logic", Duke Journal 32 (1965) 251-262. 


\section{Chapter II \\ Fundamental "Statistical. Theories} 4.

0. Introduction.

Einstein argued that since quantum mechanics is not a fundamental theory, it cannot be regarded as in any sense final. (See especially [3] and [1], on which this discussion is based.) The concept of a fundamental statistical theory may be roughly explained as follows. Let us suppose that a certain class $K$ of physical systems may be known with complete precision. For any system of this class it is possible to completely specify its type and its state. Both pieces of informatiop--the possible types of system, and their possible states--are theory relative. What is assumed is that for any $S$ in $K$ there is no extra-theoretical limit on the amount of information obtairable concerning $S$. I shall say that a statistical theory is fundamental if it is based on a maximal amount of information concerning the systems of $\mathrm{K}$. That is to say, for a fundamental theory, the degree of imprecision of our knowledge may be ignored, since the theory is supposed to hold even when this is made arbitrarily small.' By contrast, a/statistical theory which is not fundamental, is explicitly designed to take account of the case where, for whatever reasons, a maximal amount of information is not available.

For example, in classical statistical mechanics, the theoretically important states are cháracterized by some positive dispersion. In this case the dispersion is easily 
explained in terms of the incompleteness of our knowledge of the exact phase poirt of the system. The fundamental theory is given by classical mechanics which represents the time evolution of the phase point of the system, and the possible phase points are in one-to-one correspondence with dispersion free states; these are the pure statistical states of classical mechanics.

The pure statistical states of the quantum theory are not dispersion free. In this sense, the theory is significantly statistical. The problem with which this paper is concerned is, Under what conditions is a significantly - statistical theory correctly regarded as fundamental? $+\quad$ - In the case of atomic systems, the response to this. question favored by many physicists consists in denying that any theory can be fundamental in the sense just outlined. (Cf. e.g. Pauli's letters 115 and 116 to. Born in [1] as well as the subsequent commentary by Born.) Knowledge of the systems dealt with by the quantum theory is essentially.in- complete in the sense that any predictively adequate theory $\Rightarrow$ must accept the existence of a significant restriction on what can be known concerning this class of systems. Begining with Heisenberg's $\gamma$-ray microscope thought experiment; there is a long series of quasi-physical arguments aimed at making this view plausible. All of these arguments appeal to the operational incompatibility of 0 direct measurements of certain pairs of physical magnitudes. This is quite irrelevant as* Einstein showed. His argument 
may be briefly reconstructed as follows.

Two systems $S_{1}$ and $S_{2}$ are coupled if there exist magnitudes $A_{i}^{l}$ and $A_{j}^{2}$ af $S_{1}$ and $S_{2}$ (respectively) such that the probability that $A_{i}^{l}=\lambda_{i}$ is $I(0)$ if and only if the probability that $A_{j}^{2}=\lambda_{j}$ is $0(1)$. (It. is a theorem that any two quantum mechanical systems which interact and then. separate are coupled in this sense. A classic example is given by a pain of spin-1/2 particles in the singlet spin state.) In any theory admitting the existence of coupled systems, it is unnecessary to interact directly with $S_{1}$, say, in order to determine the value of the magnitude $A_{i}^{l}$; it suffices to measure the magnitude $A_{j}^{2}$ of $S_{2}$. Since the systems are spacially separated, this cannot possibly affect $S_{1}$.

The fact that quantum mechanics admits the existence of coupled systems mearis that the theory does not support the usual (operationist) interpretation of the statistical character of the theory". The idea that our knowledge is essentially incomplete assumes that a direct measurement of all: magnitudes is not possible. This of course may well" be true. The diffieulty is that direct measurements are not necessary for determining the values of the $A_{i}^{1}$; moreover, this fact is a consequence of the quantum theory. Einstein made a definitive contribution to this phase of the problem by showing that the rejection of verificationism removes any methodological objection to fundamental theories of atomic systems. 
The solution developed in this paper is that a statistical theory is fundamental only if it is gomplete; moreover the quantum theory is complete. Hence, moves $a_{\text {further }}$ objection to regarding quantum mechanics as a fundamental theory. Clearly the major problem with this approach is that completeness is apparently inconsistent . . with the significantly statistical character of the quantum theory.

The account of completeness presented in Section 1 is based on a critical analysis of Bub's very important work, "On the Completeness of Quantum Mechanics".. This paper assumes familiarity with the concepts and theorems of [7].

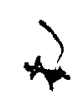

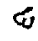


1. The Completeness of Statistical Theories.

Let us consider the statistical theory of a fixed system S. Such a theory consists of an algebra $\underline{M}$ of physical magnitudes ( $i t$ is assumed that $\underline{M}$ is at least a partial algebra) together with a set $\underline{S}$ of statistical states. Elements $\psi \varepsilon \underline{S}$ assign probabilities to ranges of values of magnitudes in $M$ : for each $A \varepsilon M$, and $\psi \varepsilon \underline{S},{ }_{A, \psi}(U)$ denotes the probability that the value of the magnitude $A$ lies in the (measurable) subset $U$ of Real numbers. $\left(P_{A, \psi}: \underline{F}(R)+[0,1]\right.$ is the distribution function of the magnitude A determined by $\psi$. )

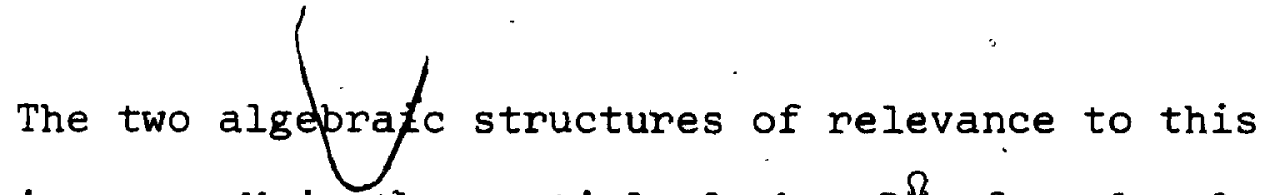
discussion are: $M$ is the partial algebra $R^{\Omega}$ of real valued functions on a classical phase space. $M$ is the partial algebra $\underline{N}(H)$ of self-adjoint operators on a separable Hilbert space. These correspond, respectively, to classical and quantum mechanics. Compatibility is interpreted as commutativity: For $A_{1}, A_{2}$ in $M_{1}, A_{1} \leftrightarrow A_{2}$ if and only if $A_{1} A_{2}=A_{2} A_{1}$. It is a theorem that $A_{1}$ and $A_{2}$ commute if and only if there exts a $C \varepsilon M$ afd Borel functions $g_{i}: R \rightarrow R$ $(i=1,2)$ such that $A_{i}=g_{i}(C)$. Linear sums and products of compatible magnitudes may be defined by the linear sum and product of the associated functions $g_{i}$. Explicitly:

$$
\lambda_{1} A_{1}+\lambda_{2} A_{2}=\left(\lambda_{1} g_{1}+\lambda_{2} g_{2}\right)(C)
$$

$\left(\lambda_{1}, \lambda_{2} \varepsilon R\right)$ and

$$
A_{1} A_{2}=\left(g_{1} g_{2}\right)(C) .
$$


The subalgebra of idempotent elements of $\underline{M}$ form a partial Boolean algebra $L$. In the case of $R^{\Omega}$. this is the subalgebra $B$ of characteristic functions on $\Omega$. This is - isomorphic to the field $\underline{F}(\Omega)$ of (measurable) subsets. of a. For $\mathrm{N}(\mathrm{H})$, this is the partial Boolean algebra $\mathrm{N}$ of projection operators. $\mathrm{N}$ is isomorphic to the partial Boolean algebra $B(H)$ of closed linear subspaces of $H$. [Let $u, n, '$ denote the spans intersection, and orthocomplement of the subspaces in $H$. Then for $a, b$, in $H, a+b$ if there exist mutually orthogonal subspaces $a_{1}, b_{1}, c$ súch that $a=a_{1} v c$ and $b=b_{1} u c$. The operations $u, n$ are restricted to $\leftrightarrow$. $H$ is the unit of $B(H)$ and $\{0\}$ is the 0 of $B(H)$. Physical properties are introduced in terms of the magnitudes $A$ in $M$ as follows. Take a real number $\lambda$ in the range of $A$. Then $A=\lambda$ (this is read "the value of $A$ is $\lambda "$ ) is a property of $S$. More generally, given a subset $U$. of $R$ and magnitude $A, A \varepsilon U$-- the value of $A$ lies in $U$-- represents a property of the system $S$.

Von Neumann ([9] Ch. III. 5) observed that every i. property of $\mathrm{S}$ is mepresented by an idemptent magnitude in L. This is simply seen in the case of classical mechanics. Let $\Omega$ be a subset of $n$-dimensional Euclidian space. An ." elementary event in the history of $\mathrm{S}$. is represented by a point in 凡. 'As is wezl-known, an event $\omega$ is associated with a pure statistical state of classical mechanics: the 2-valued measure on $\underline{F}(\Omega)$ determined by $w$. Now let $f_{A}$ be a real valued function in $R^{\Omega}$ representing the magnitude $A$. A 
property $A \varepsilon U$ holds for $S$ if and only if $S$ is in a state $\omega$ such that $f_{A}(\omega) \varepsilon U$. - Let $\Gamma$ denote the subset $f_{A}^{-1}(U)$ of $\Omega$. It is clear that $S$ has the property $A \varepsilon U$ if and only if the state $\omega$ of $S$ lies in $\Gamma$. The property $A \in U$ is said to be associated 'with $\Gamma$. 'In general there are many properties $A_{i} \varepsilon U_{j}$ such that $f_{A_{i}}^{-1}\left(U_{j}\right)=\Gamma$ for some $U_{j} \in R$. Now let a be the characteristic function of $\Gamma$, and let, $P$ be a propert associated with $\Gamma$. By the correspondence between properties of $S$ and subsets of $\Omega_{3}$ it follows that every pro- ${ }^{\prime}$ perty $P$ is represented by the characteristic function $a$, in the sense that $P$ holds if and only if $S$ is in a state $w$ such that $a(\omega)=1$. Since $a$ is two valued this is equivalent to $a(\omega), \neq 0$.

The situation in quantum mechanics is exactly analogous. Elementary events, represented by rays $\underline{K}$ in $H$, are associated with pure statistical states. In quantum mechanics statistical states are given by measures on the closed linear subspaces of $\mathrm{H}$. The pure state associated with $\underline{K}$ is determined by taking the square of the norm of the projection of a unit vector lying in $\underline{\underline{k}}$ onto each subspace of $\mathrm{H}$. Since there is a one-to-one correspondence which associates each projection operator with the subspace which is its range, this determines a probability measure on $\mathrm{N}$. Recall atoms in $B$ are characteristic functions of singleton subsets $\{\omega\}$ of $\underline{F}(\Omega) . \quad N$ is also atomic. An atom in $N$ is a projection operator onto a one-dimensional subspace of. $H$. Thus in each theory, there is a one-tolone correspondence between elementary 
events and atoms in $\underline{L}$.

To summarize: Every magnitude may be replaced by' a: set of properties, and every property corresponds to a twovalued quantity, i.e. to an idempotent magnitude. (For this reason it suffices to consider the algebra $L$ of idempotent " magnitudes.) The correspondence is not one-to-one since $\because$ very many properties are associated with the same subset of $\Omega$ (or subspace of $\mathrm{H}$ ), and therefore, represented by the same idempotent magnitude. There is a one-one correspondence between idempotent magnitudes and equivalence classes of properties represented by the same idempotent in $\underline{L}$. An idempotent may therefore be thought of as the equivalence class of properties it represents.

For any statistical theory, we may distinguish two. ways of viewing the algebra of idempotent magnitudes. First, L may be .regarded as an abstract property of physical magnitudes: in this case, the idempotents in $M_{3}$ are simply postulated as having the structure $\underline{L}$. The algebraic structure intfoduced in this way is termed the logice(l space $\underline{L}_{1}$ of a statistical theory. (Bub [2] p. 45.) The characteristic feature of $\underline{L}_{1}$ is that its introduction is independent of statistical considerations.

There is another way of viewing the algebraic structure of a statistical theory: Let $M$ be trieset of physical magnitudes: No algebraic structure is, assumed for $M$. Rather an algebraic structure is defined in terms of the distribution functions $P_{A, \psi}$ of the magnitudes $A \varepsilon M$ by writing $A_{1} \leftrightarrow A_{2}$ 
if there is a $C \varepsilon M$ and functions $g_{i}: R \rightarrow R(i=1,2)$ such that

$$
P_{A_{i}, \psi}(U)=P_{C, \psi} g_{i}^{-1}(U)
$$

for all $U \subseteq R$ and $\psi \in \underline{S}$. I.e. the magnitudes $A_{1}$ and $A_{2}$ are compatible if they are statistically equivalent to $g_{i}(C)$. The definition of the partial operations is given in terms of the associated functions as before. The resulting structure $\underline{M}_{2}$ is determined by the set $\underline{S}$ of statistical states of the theory. The subalgebra of idempotents of $\underline{M}_{2}$ is termed ([2] p. 45) the Iogical space $\underline{L}_{2}$; it is distinguished from $\underline{L}_{1}$ by. its dependence on the relation of statistical equivalencè.

It is important to recognize that the elements of $\underline{L}_{1}$ and $\underline{L}_{2}$ are the same. Each logical space consists of the subset of idempotent magnitudes in $M$ or the equivalence classes of properties which they represent. Since $\underline{L}_{1}$ and $\underline{L}_{2}$ are independently specified, they may be structurally different. Here it is essential to be very clear: Although the relation. of statistical equivalence is obviously an equivalence relation on $\underline{L}_{1}$, it is not necessarily compatible with the structure of $\underline{L}_{1}$, so that $\underline{L}_{1}$ and $\underline{L}_{2}$ may not even be homomorphic.

Within this framework, Bub has proposed a general criterion for the completepess of statistical theories: A statistical theory is said to be complete if and only if the logical spaces $\underline{L}_{1}$ and $\underline{L}_{2}$ are isomorphic ([2] $\left.\dot{p} \cdot 45\right)$ ). The problem of demonstrating the isomorphism of the two logicalspaces is the completeness problem for a statistical 
theory." Gleason's theorem [4] establishes that all generalized probability measures on $B(H)$ afe given by the statistical agorithm of quantum mechanics. "(Here it is necessary" , to assume, that $H$ is at least three dimensional.) Gleason's theorem is regarded as having sol the completeness problem for quantum mechanies in this sense.

Now the first point to notice is that the isomorphism condition is, ip'most cáses, automaticaliy satisfied. So far as any actually proposed statistical theory is concerned, $I_{1}$ is defined as $\underline{L}_{2}$. But if this is the dase, the completenèss problem is trivial: It might be argueq that intro-. ducing $L_{1}$ in this way obscures the fact that it is always. possible to constructi a statistical theory where the isomorphișm condition does ${ }^{\circ}$ not hold. A construction of this type amounts to a reinterpretation of the statistical theory; in the case of the quantum theory, hidden variable theories may be viewed as reinterpretations in this sense. It would appear that the isomorphism gondition is intended to exclude" a réinterpretation based on a structurally different $\underline{L}_{1}$ on the ground that such:a theory is incomplete. This suggests that the isomorphism cóndition is of the greatest importance, when considering theories of this type what is Anclear is that hidden variable reinterpretations are unsatisfactory because they are incomplete. I will return to this question in a.moment. At this point I want to examine. the case $q$ theories for which the relation of "statistical equivalence" is compatible" with the operations and relations of $\underline{L}_{7}$. 
A set of statistical states on $\underline{L}_{1}$ is full if and only if it is order-determining. I.e. if $\psi(a) \leq \psi(\hat{b})$ for all $\psi \varepsilon \underline{S}$ implies $a \leq b, a, b$ in $\underline{L}_{1}$. Clearly, if statistical equivalence is compatible with $\underline{L}_{1}$, then $\mathrm{L}_{1}$ and $\mathrm{L}_{2}$ are isomorphic if and only if $\dot{A}^{*}$ is a full set of statistical states.

It is not thial $\underline{L}_{1}$ has a fuli set of states. Ther even exist pärtial Boolean algebras which admit no states. (See Greechie and Gudder [5] Sect. 7 for a discussion and references.) The difficulty is that even if it. can be shown that $\underline{S}$ is full on $\underline{L}_{1}$, and hence that $\underline{L}_{1}$ and $\underline{L}_{2}$ are isomorphic, the existence of possible extensions of the theory would remain an open question. An extension is defined as follows: Let ha $\underline{L}_{1} \rightarrow \underline{L}_{1}^{\prime}$ be a homomorphism between the two partial Boolean algebras " $\underline{L}_{1}$ and $\underline{L}_{1}$. Let $\underline{S}$ and $\underline{S}^{\prime}$ derote the associated sets of statistical states. Then $\psi^{\prime} \varepsilon \underline{S}$ ' is an extension of $\psi \varepsilon \underline{S}$ if $\psi^{\prime}=\psi^{\prime} \circ h^{\prime}$. $\underline{S}^{\prime}$ is an extension of $\underline{S}$ if every $\psi \varepsilon \underline{S}$ has 'an extension in ' $\underline{S}^{\prime}$. The statistical theory $\left(\underline{L}_{1}^{\prime}, \underline{S}^{\prime}\right)$ is an extension of the theory $\left(\underline{L}_{1}, \underline{S}\right)$ if $\underline{S}^{\prime}$ is an extension of $S$ and $h$ is an imbedding. The extension is proper if for someo $\left.\psi_{s}^{\prime} \varepsilon, \underline{s}^{\prime}, \psi^{\prime} \mid \mathrm{h}^{\hat{q}} \underline{L}_{1}\right] \neq \psi$.

$\therefore \quad$ Now Gleason's' theorem excludes certain extensions of the quantum theory. For logical spaces represented by $B(H)$, Gleason's theorem is equivalent to the general result that the quantum theory has no proper extensions in the category of partial Boolean algebras. A Boolean extension may be defined as an extension for which $L_{1}^{\prime}$ is a Boolean algebra. 
Theorems 0 and 1 of [7] imply that the quantum theory has no Boolean extensions. This is a similar (but weaker) result concerning \& sub-category of the category of partial Boolean algebras.

It is claimed that the isomorphism condition fully explicates the sense in which Gleason's theorem may be regarded as a completeness theorem for quantum mechanics. But the existence of extensions is simply ignored by this condition, since isomorphism concerns only the structures $\underline{L}_{1}$ and $\underline{L}_{2}$. A priori, there is no reason to expect that completeness in this sense is incompatible with the existence of imbeddings of $\underline{L}_{1}$, leading to extensions of the theory. This is a defect, since an analysis of completeness in quantum mechanics "should capture the full scope of the problem solved by Gleasón.

This is perhaps more clearly a difficulty for an analysis which explicitly treats completeness as the isomorphism of two compatible logical spaces. I say this because if Bub intends completeness to apply only to those statis- tical theories for'which statistical equivalence is compatible with the structure of $\underline{L}_{1}$; then the whole motivation for regarding $\dot{L}_{1}$ and $\underline{L}_{2}$ as independent logical spaces becomes obscure. This distinction seems basic to the whole analysis. The difficulty is that it is not especially? relevant to at least one phase of the Completeness problem; it fails, to provide an explication of the mathematical problem solved by Gieason's theorem and its corrollaries. To put this : 
slightly differently: the characterization of $\underline{L}_{1}$ and $\underline{L}_{2}$ leads to two independent logical spaces; the isomorphism condition establishes a connection between the two structures. The problem is, how are we to regard this connection?

Notice that $\underline{L}_{2}$ is essentially the logical space determined by infinitely precise measurements performed on infinitely many copies of $\mathrm{S}$. $\underline{\mathrm{L}}_{2}$ is explained by the assump"tion of an underlying logical structure $\underline{L}_{1}$. In other words, $\underline{L}_{2}$ forms the ideal evidential basis for the hypothesis that the algebraic structure of $L$ is given by $\underline{L}_{1}$, and the isgmorphism condition is just the simplest restriction on the structure of $\underline{L}_{1}$ which is consistent with the structure determined by $\underline{L}_{2}$. The important point which the distinction between $L_{1}$ and $L_{2}$ clarifies is that $\underline{L}_{1}$ represents a structural assump fion which occurs quite explicitly in the derivation of the (ideal) statistical predictions of the theory. The problem of completeness raises a further question,viz., Does the algebraic structure determined by the isomorphism condition occur essentially, or is it possible to regard it as merely a property of a particular formulation of the , theory?

The discussion of Gleason's theorem suggests that a statistical theory $\left(\underline{L}_{1}, \underline{s}\right)$ is complete if it has no proper extensions. Completeness in this sense is compatible with the fact that the quantum theory does not contain dispersionfree states: If the logical space $\underline{L}_{1}$ were imbeddable into a Boolean algebra; quantum mechanics would be an incomplete 
theory, since by the fundamental property of Boolean algebras, $\underline{L}_{1}$ must admit two-valued measures. But there exist nonBoolean logical spaces, represented fy $B(H)$. For this class of logical spaces, Gleason's theorem implies that the quantum theory is complete, even though $\underline{S}$ does not contain two'-valued measures.

This analysis assumes that completeness is relative to a cafegony of algebraic structures. It might be objected that this amounts to an unnecessary weakening of the concept of completeness. Intuitively it may seem that a statistical theory is complete only if it contains all statistical states. In other words, completeness should depend only on the membership of $\underline{S}$, so that a statistical theory is incomplete if it fails to include dispersion free states.r

The first point to notice is that this' suggestion only appears to make completenéssizindependent of some underlying notion of algebraic structure. If a theory must contain dispersion free states, the only complete theories - are those for which $\mathrm{L}_{1}$ may be homomorphically mapped into a Boolean algebra.. (This is basically a consequence of Theorem 0 of [7].) The inclusion of additional states therefore limits the class of logical structures compatible with the completeness of a statistical theory to the category of partial Boolean algebras which are homomorphically related to a Bọolean algebra. (See [7] Sect. 5 for a definition of this concept.) Every logical space of a complete 
theory is represented by an object of this category. From the point of view of possible extensions of a statistical theory, this modification results in a mathematically, weaker concept of completeness. The category of partial Boolean algebras which are homomorphically related to a Boolean algebra is a proper subcategory of the category of all partial Boolean algebras. So the completeness problem is limited to showing that there are no extensions in this sub-category.

Secondly, this objection has a certain initial plausibility when it is implicitly assumed that the nonexistence of two-valued homomorphisms has the same significance in both imbeddable and non-imbeddable structures. In the case where $\underline{L}_{1}$ is imbeddable into a Boolean algebra, distinctions are possible which are obscured by the absence of dispersion free states. E.g. this is true of the nonatomic lattice of idempotent macroscopic magnitudes of classical statistical mechanics. Now the case of a nonimbeddable $\underline{L}_{1}$ is very different, $N$ contains pairs of idempotents such that $a \cdot \neq b$ but $h(a)=h(b)$ for every homomorphism $h: \mathrm{N} \rightarrow \mathrm{Z}_{2}$. Thus a and $\mathrm{b}$ are distinguishable quantum mechanically, but not classically, i.e. not in țerms of a homomorphism ònto, $z_{2}$ ( = the two element Boolean algebra).

To summarize this discussion of [2]: The distinction between $\underline{L}_{1}$ and $\underline{L}_{2}$ clarifies" the fact that the introduction. of an algebra of idempotents represents an additional ex- 
planatory assumption of a statistical theory. This structure occurs essentially in determining the set of statistical states of the theory. In, the case of quantum mechanics, the nature of this assumption was first made completely explicit by von Neumann. Completeness means that $L_{1}$ has no proper extehsions. The quantum theory is complete with respect to the category of partial Boolean algebras. This is the content of Gleason's theorem. By Stone's representation theorem (on ore accurately, by the representation theorem for Boolean $\sigma$-algebras of Loomis and Sikorski), classical mechanics is complete relative to the category of Boolean algebras. (Since this makes the theory of states on a Boolean algebra a sub-theory of the theory of measures on an arbitrary field of sets.) It is in this sense -the sathe sense in both cases -- that quantum mechanics and classical mèchanics are complete statistical theories.

$\therefore$ One final remark concerning the distinction between $\underline{L}_{1}$ and $\mathrm{L}_{2}$ : There is a similarly named, but otherwise very different distinction given by van Fraassen. In [8] elements of $L_{1}$ are theoretical statements; they correspond to what I called properties. For van Fraassen $L_{1}$ is a set of objects which is not necessarily related to the algebra of idempotent magnitudes of the theory. Elements of $\mathrm{L}_{2}$ are statements of the form: "The value of $\backslash A$ is certainly in $U$ " or "The value of A lies in $U$ with probability $\lambda \varepsilon[0,1] "$. Van Fraassen's $E_{1}$ and $\mathrm{L}_{2}$ are distinguished by their elements; $\mathrm{L}_{1}$ and $\mathrm{L}_{2}$ consist of essentially different statements. Moreover no structure is 
assumed for $L_{1}$. Two algebraic structures are imposed on the statements of $\mathrm{L}_{1}$. (Cf. [8] Sections II.4 and II.5.) Each of these is based on the set. $\underline{S}$ of statistical states of the theory, and therefore correspond to logical spaces of the type $\underline{\mathrm{L}}_{2}$.

For van Fraassen, logical structures occur only at the level of the evidential basis of a statistical theory, and never as explanatory principles. This is a distortion of what actually occurs in either clajssical on quantum mechanics. In both cases the algebraic structure introduced by $\mathrm{L}_{1}$ occurs explicitly and essentially in the assignment of probabilities to the ranges of values of the magnitudes of the theory. This is the principle introduced by quantum mechanics which leads to the significantly statistical character of the theory. The fact that $\underline{L}_{1}$ is not imbeddable into a logical space which admits two-valued measures shows that the statistical character. of the theory is an essential (component of this assumption and supports the view that quantum mechanics is a fundamental theory. 


\section{REFERENCES}

[1] M. Born, The Barn-Einstein Letters: The Correspondence

Between Albert Einstein and Max and Hedwig Born: 1916-1955, Walker and Company, 1971:

[2] J. Bub, "On the Completeness of Quantum Mechanics" in Contemporary Research in the Foundations and Philosophy of Quantum The fry, ed. by C. Hooker, Reidel, 1974.

[3] A. Einstein, "Quantum Mechanics and Reality" (1948), in $[1], 168-173^{\circ}$.

[4] A: Gleason, "Measures on the Closed Śbspaces of Hilbert Space", Journal of Mathematics and Mechanics 6 (1957) $885-893$

[5] R. J. Greechie and S. Gudder, "Quantum Logics" in Contemporary Research in the Foundations and Philosophy of Quantum Theory, ed. by C. Hooker, Reidel 1974.

[6] S. Gudder? "A Generalized Measurfe and Probability Theory for thk Physical Sciences", in Fdundations of Probability, Statistical Inference and Statistical Theories of Science, ed. by C. Hooker and W. Harper, Reidel (forthcoming).

[7] S. Kochen and E. P. Specker, "The Problem of Hidden Variables ín Quantum Mechanics", Journal of Mathematics and Mechanics 17 (1967) 59-87.

[8] B. van Fraassen, "Semantic Analysis of Quantum Logic" in Contemporary Research in the Foundations and Philosophy of Quantum. Theory, ed. by C. Hooker, Reidel, 1974. 
[9] J. von Neumann, Mathematical Foundations of Quantum Mechanics, Princeton University Press, 195.5. 
Chapter III

The Possibility Structure of Physic̈al Systems

0. Introduction.

This paper develops the logical interpretation of nor-relativistic quántum mechanics initially proposed by. Hilary. Putnam [11]. YSee also [1] and [2].) The main features of this interpretation are briefly summarized in. this introduction.

Certain physical theories postulate abstract structural constraints which events are held tq satisfy. Such theories are termed "principle theories". Interpretations of principle theories aim to explain their relation to the theories they replace. Interpretations are therefore concerned with the nature of the transitions between theories.

Theories of space-time structure provide the most accessible illustration of principle theories. For, example, Newtonian mechanics in the absence of gravitation represents the 4dimensional geometry of space-time by the inhomogeneous Galilean group, which acts transitively in the class of free motions, i.e. the inhomogeneous. Galilean group is the symmetry group of the free motions: it is a subgroup of the symmetry group of every mechanical system, and the largest such subgroup. Einstein's special principle of relativity is the hypothesis that the symmetry group of the free motions is the Poincare group. The transition from the Galilean group to the Poincare group is associated with a corresponding modification in space- 
time structure. The absolute time and Euclidean metric. of Newtonian. mechanics are dropped altogether, and the metrical relations of space-time are determined by the Minkowski tensor.

The special theory of-relativity represents the transition from Newtonian Mechanics to Maxwell's electrodynamics as involving a modification of the structure of space-time. In this sense, the special theory may be regarded as an interpretation of classical electrodynamics.

Theoretical transitions in the class of space-time theories suggest an analogous approach to the interpretation of quanţum mechanics. In this view, classical and quantum mechanics are"represented as $a^{\prime \prime}$ particular type of principlé theory. I call theories of this type "theories of logical structure" ( or sometimes "phase space theories"), since the type of structural constraint they introduce concerns the logical structure of events and this is given by the algebra of idempotent magnitudes of the theory. The logical structure of a physical system imposes the most general kind of constraint on the occurrence and non-occurrence of events. The event ${ }^{\circ}$ structures of classical mechanics are essentially Boolean algebras. The logical structure of a quantum mechanical system is represented by the partial Boolean algebra' of subspaces of a Hilbert space. In general, this is not imbeddable into a Boolean algebra.

The mathematical investigations of Kochen and Specker [9] lead to a general concept of completeness applicable to 
phase space theories. The explication depends on the notion of a proper extension of a phase space theory. 'Extensions are defined relative to a category of algebraic structures (representing the phase spaces of the theory) and a suitable concept of statistical state: Let A demote the partial Boolean algebra of idempotents, $\underline{S}$ the sét of statistical states $\psi$ on A. Suppose there is an imbedding $\phi$ carrying A into $A^{\prime}$ such that for every $\psi \varepsilon \underline{S}, \psi=\psi^{\prime} \circ \phi$, where $\psi^{\prime \prime}$ is a statistical state on $A^{\prime}$. Then the theory $\left(A^{\prime}, \underline{S}^{\prime}\right)$ is an extension of the theory $(A, \underline{S})$. The exterision is proper if for pome $\psi^{\prime} \varepsilon S^{\prime}$, $\psi^{\prime} \mid \phi[\mathrm{A}] \neq \psi$. Complete phase space theories have no proper extensions.

A proper" extension of a phase space theory, must, not be confused with the more usual notion of a proper extension of a formal theory. Besides trivializing the notion, this would imply that completeness is a-propenty of the theory's formalization. This, however, is not the caqe, The relevant notion of completeness is a mathematical property of a certain class of algebraic structures rather than a metamathematical one. There exists an important connection between completerless and the formal theory of this class of structures, but the concept of completeness does not depend on this connection.

A great deal of unclarity has surrounded the problem of completeness in quantum mechanics. An important consequence of this 'analysis is that classical mechanics and quan'tum mechanaics are complete in'exactly" the same sense. In neither theory do there exist extensions in the category of algebric structures Bsociated with their respective phase spaces.'. As'. principle theories, ckassical mechanics and quantum méchanics 


\section{3}

specify different kinds of constraints on the possible events open to a physical oystem, i.e. they determine different possibility structures of events, and each theory is complete relative to the category of algebraic structures defined.

Finally, the approach to phase space theories outlined here has interesting consequences for the nature of lqgical truth. The logical structures of quantum mechanics include the Boolean algebras of classical mechanics.' Such structures represent the possibility structure of events, that is, roughly speaking, they represent the-way in which the properties of a physical system hang together. The quantun theory has shown that significantly different assumptions may be made concerning this structure. Now classical propositional validity is essentially validity in the category $B$ of general. Boolean algebras. The choice of Boolean algebras, has an empirical justification in classical mechanics, for the magnitudes of this theory form a commutative algebra 'and therefore the subalgebra of idempotent magnitudes form a Boolean algebra.? When viewed in this way, the justification of classical validity is infimately bound up with the alogicai structures postulated by classical mechanics. The quantum theory extends this class of structures to include all partial Boolean algebras' of a certain type. The Boolean imbedda- Dility properties of these structures have a model-theoretic characterization in terms of the validity of classical tau-, toiogie. Now a consequence of the work of Koonen and Specker is that there exists a classical tautology which is quantum 
mechanically refutable (ie. refutable in a partial Boolean algebra of the quantum theory). In this sense, classical logic is false, and the truth of logic, an expirjeal questimon.

[One remark on the mathematical exposition: All

" qualifications regarding mensurability; viz.; the restrictLion to Bore functions, Bore subset's; and Boolean $\sigma$-algabras, have been omitted. This merely means that the expos-. ion is not. as general as it might o ge. $]^{\circ}$ 
I.. Preliminary Notions:'

A partial algebra over a field $K$ is a set $A$ with a reflexive and symmetric binary relation $\leftrightarrow$ (termed "compatibility") such that $A$ is closed under the operation of scalar multiplication from $K \times A$ to $A$, and the operations of addition and multiplication defined from $\leftrightarrow$ to $A$. That is:

(i) $\leftrightarrow \subseteq \mathrm{A} \times \mathrm{A}$

(ii) every element of $A$ is compatible with itself

'(iii) if a is compatible with bp then b is compatible with a, for all $a, b \varepsilon A$

(iv) If any $a, b, c \varepsilon A$ are mutually compatible, then $(a+b)++c$, $a b \leftrightarrow c$, and $\lambda a \leftrightarrow b$ for all $\lambda \in k$.

In addition, there is a unit element 1 which is compatible with.every element of $A$, and if $a, b, c$ are mutually compatible, to

then the values of the polynomials in $a, b, c$ form a commuta$\therefore \therefore$.

tive:algebra over the field $\mathrm{K}$.

$\because$ A partial algebra over the field $\mathrm{z}_{2}$ of two elements is termed a partial Boolean algebra. The Boolean operations $\wedge, v$ and ' may be' defined in terms of the ring operations in the usual way:

$$
\begin{aligned}
& a \wedge b=a b \\
& a \vee b=a+b-a b \\
& a^{\prime}=1-a .
\end{aligned}
$$

If $a, b, c$ are mutually compatible, then the values of the : polynomials in $a, b, c$, form a Boolean algebra.

"Clearly, if $B$ is a set of mutually, compatible elements.in a partial algebra $A$, then $B$ generates a commuta-. tive sub-algebra in $A$; and in the case of a partial Boolean algebra 
$A$, 'B generates a Boolean sub-algebra in $A$. Just as the set of idempotent elements of a commutative algebra forms a Boolean algebra, so the set of idempotents, of a partial algebra forms a partial Boolean algebra.:

We shall be mainly concerned with partial* Boolean algebras. A homomorphism, $h$, between two partial Boolean algebras, $A$ and $A^{\prime}$, is a map $h: A+A^{\prime}$ which preserves the algebraic operations, i.e. for all compatible a, beA:

$$
\begin{aligned}
& h(a) \leftrightarrow h(b) \\
& h(a+b)=h(a)+h(b) \\
& h(a b)=h(a) h(b) \\
& h(1)=1
\end{aligned}
$$

"A homomorphism is an imbedding if it is one-to-one.

A weak imbedding is a homomorphism which is an imbedying on Boolean sub-algebras of A: More precisedly, a homomorplism; $h$, of $A$ into $A^{\prime}$ is a weak imbedding if $h(a) \neq$ $h(b)$ whenever $a+b$ and $a \neq b$ in $A$. So that in the case of a weak imbedding, incompatible elements may be mapped onto the same element.

An algebra is simple if its only proper filter is . the unit filter \{1\}. $z_{2}$ is the oniy simple Boolean algebra. A necessary and sufficient condition for the imbeddability of a partial Booleap algebra A f pto a Boolean algebra $B$, is that for every pair of distinct lements $a$, beA there exists a homomorphism $h: A \rightarrow Z_{2}$ which separates them in $Z_{2}$, i.e. such that $y(a) \neq h(b)$ in $z_{2}$. This is Kochen and specker's Theorem 0 . The result depends on the semi-simplicity pro-: 
perty of Boolean algebras, i.e. the fact that every Boolean algebra is imbeddable into a direct union of the simple Boolean algebra $\mathrm{Z}_{2}$.

The direct union of a family $\left\{B_{i}\right\}_{i \in I}$ of Boolean algebras is defined on the set of all sequences $\left\{a_{i}\right\}_{i \in I}$ of elements of the $B_{i}$. The operations are defined point-wise, i.e.

$$
\begin{aligned}
& \left\{a_{i}\right\}_{i \in I}^{\prime}=\left\{a_{i}^{\prime}\right\}_{i \varepsilon I} \\
& \left\{a_{i}\right\}_{i \varepsilon I^{\vee}\left\{b_{i}\right\}_{i \varepsilon I}=\left\{a_{i} \vee b_{i}\right\}_{i \varepsilon I}} \\
& \left\{a_{i}\right\}_{i \varepsilon I} \wedge\left\{b_{i}\right\}_{i \varepsilon I}=\left\{a_{i} \wedge b_{i}\right\}_{i \varepsilon I . "}
\end{aligned}
$$

The direct product of the $B_{i}$ is essentially the closure of the direct union under the operation of forming isomorphic images.

Semi-simplicity is equivalent to the homomorphism theorem: Every Boolean algebra admits a two-valued homo- a morphism, i.e. a homomorphism onto $z_{2}$.

The semi-simplicity property and the homomorphism theorem are alternative formulations of Stone's representation theorem and the ultrafilter theorem (respectively). This is a consequence of the fact that in every Boolean algebra there is a natural one-to-one correspondence between ultrafilters and two-valued homomorphisms. Let $S$ be the Stone space of a Boolean algebra $B$. " ( $S$ is the set of all ultrafilters in $B)$. Let $P(S)$. denote the Boolean algebra of all subsets of $S$. Replacing ultrafilters by two-valued homomorphișms and subsets of $S$ by the sequence of yalueg of 
their characteristic functions yields $z_{2}^{S}$-- the direct union of $z_{2}$ to the power of $S$-- from $\underline{P}(S)$. In this context, the

Stone isomorphism becomes the imbedding $k: B \rightarrow Z_{2}^{S}$ given by

$$
k(a)=\left\{h_{t}(a)\right\}_{t \varepsilon S} \text {. }
$$

The mathematical connection of these ideas to logic ardes in the following way. Propositional formulae are regarded as Boolean polynomials in a suitable first-order language $\underline{L}$. Realizations of $\underline{L}$ are objects in the category $B$ of general Boolean algebras. A formula $\phi\left(x_{1}, \ldots, x_{n}\right)$ is - classically valid ( $C$-valid) if, for any $B$ in。B every substitution of elements for the variables $x_{1}, \ldots, x_{n}$ yields the unit of $B$. If $\phi$ is a propositional formula not valid in $B$, i.e. if $\phi(a) \neq 1$ for some $a=\left(a_{1}, \ldots, a_{n}\right)$ in $B^{n}$, then $\phi(k(a)) \neq 1$ in $z_{2}^{S}$, where $k(a)=\left(k\left(a_{1}\right), \ldots, k\left(a_{n}\right)\right)$, so that $\phi$ is refutable in $Z_{2}$. Hence, by semi-simplicity, classical. validity is equivalent' to tautologousness, i.e. validity in $\mathrm{Z}_{2}$.

Now extend the class of rearizations of $\underline{L}$ to the category of partial Boolean algebras. Validity in a partial Boolean algebra $N$ depends on the domain of a propositional formula. $\phi\left(x_{1}, \ldots, x_{n}\right)$ is valid in $N$ if every substitution of elements from the domain of $\phi$ yields the unit of $N$. The concept of the domain of a propositional formula may be simply explained by an example.

Let $\phi=\psi \equiv x$ be the propositional formula 


$$
" x_{1} \wedge\left(x_{2} \vee x_{3}\right) \equiv\left(x_{1} \wedge x_{2}\right) \vee\left(x_{1} \wedge x_{3}\right)
$$

The domain of $\phi$ is the set of all elements $a=\left(a_{1}, a_{2}, a_{3}\right)$ of $\mathrm{N}$ such that:

$$
\begin{aligned}
& a_{1} \leftrightarrow a_{2} \\
& a_{1} \leftrightarrow a_{3} \\
& a_{2} \rightarrow a_{3} \\
& a_{1} \leftrightarrow\left(a_{2} \vee a_{3}\right) \cdot i \\
& \left(a_{1} \wedge a_{2}\right) \leftrightarrow\left(a_{1} \wedge a_{3}\right) \\
& a_{1} \wedge\left(a_{2} \vee a_{3}\right) \leftrightarrow\left(a_{1} \wedge a_{2}\right) \vee\left(a_{2} \wedge a_{3}\right),
\end{aligned}
$$

for only then will the operations appearing in $\phi$ be defined in $\dot{N}$. t.

It follows from the first three compatibilities that any three elements in the domain of $\phi$ generate a Boolean algebra, and hence, satisfy the distributive law. Hence $\phi$ is valid in all partial Boolean algebras." [Notice also that the last three compatibilities are therefore redundant.]

The generalized definition of propositional validity .is: A propositional formula is Q-valdd if it is valid in all partial Boolean algebras. The notion of Q-validity is formalizedin [8].

The fundamental model-theoretic result, in this field is :

(i) A partial Boolean algebra $\mathrm{N}$ is imbeddable into a Boolean algebra iff for every classical tautology of the form $\phi \bar{\psi} \psi$ the corresponding identity $\phi=\psi$ is valid in $N$; i.e. $\phi(a)=\psi(a)$ holds for all a in the intersection of the 
domains of $\phi$ and $\psi$.

(ii) $\mathrm{N}$ is weakly imbeddable into Boolean algebra iff every classical tautology is valid in $\mathrm{N}$.

(iii) There is a homomorphism from $\mathrm{N}$ into a Boolean aigebra iff every classical tautology is not refutable in N. [This is-Theorem 4 of [9]. (See [2] for an exposition of the proof of this theorem.)?

Notice that both C-validity and Q-validity have been defined algebrajcally, as the validity. of propositional formulae in certain algebraic categories. In the case of classical validity, this definition differs sharply from more usual characterizations. Because of the equivalence of validity in $B$ and validity in $Z_{2}$, classical propositional validity is defined as validity in the two-element matrix or truth-table $\langle\{0,1\},\{1\}, v,->$, where $\{1\}$ is the set of "designated elements and $\mathrm{v}$ and - have their well-known matrix definitions. $\phi\left(x_{1}, \ldots, x_{n}\right)$ is a classical tautology if it yields the designated value $I$ for all substitutions of the elements 0 and $I$ for the variables $x_{1}, \ldots, x_{n}$.

The transition to Q-validity is greatly simplified when classical validity, is undenstood algebraically. But there is another reason for replacing the matrix definition. Finst, it should be clear that a definition of classical validity is not merely a stipulation. Rather, one concept (or group of concepts) proved very fruitful in initiating the modern mathematical development of logic, and a definition of validity should provide some explication of this 
concept. The matrix definition is misleading since it ignores the connection of classical propositional logic with the theory of Boolean algebras. This connection is important for the whole development of the subject. For example, on the matrix definition it is trivial that classical propositional validity is effective. But when classical validity is defined algebraically, effectiveness depends on semisimplicity, which is decidedly non-trivial. Hence the

- algebraic definition suggests that effectlveness did not play, a major role in the initial formulation of mathematical logic, and in fact, considerations of this type actually occur much later: viz., when the scope of logic came to be drawn in terms of the distinction between syntax and semantics. 
2. Partial Boolean Algebras and:Orthomodular Posets.

Every partial Boolean algebra is isomorphic to a collection $\underline{L}=\left\{L_{i}\right.$; $\left.i \varepsilon I\right\}$ of Boolean algebras satisfying the following conditions:

(i) The $L_{i}$ have a common 1 .

(ii) If a $\varepsilon L_{i} \cap L_{j}$, then $a^{t_{i}}=a^{t_{j}} c_{i}^{d_{1}}=$ ortho- complemention relative to $L_{i}$, etc.)

(iii) If $a, b \in L_{i} \cap L_{j}^{*}$, then $a \wedge_{i} b=a \wedge_{j} b$.

(iv) Let $L=u\left\{L_{i}\right.$ : iEI $\}$. Given any $a, b, c$ in $L$ such that any two of them lie in a common $L_{i}$, there exists. an $L_{j}$ suc $\ddot{B} "$ that $a, b ; c \varepsilon L_{j}$.

Families of Boolean algebras satisfying.(i) - (iv) are called logical structures. (Notice, the first three conditions simply insure that in any logical structure the operation of taking the intersection of two algebras makes. sénse.) The partial Boolean algebra associated with a logical structure is defined on $L=U L: a \leftarrow b$ iff there is an $L_{i}$ containing $a, b$. The $I$ of $L$ is the common 1 , of $a l l$ the $L_{i} \cdot a^{\perp}=a^{\perp_{i}}$ for some $L_{i}$. If $a \leftrightarrow b$, then $a \wedge b=a \wedge_{i} b$ for some iEI. (The zero and join of $L$ are thought of "' as being defined in the usual way.)

A partial Boolean, algebra is said to be transitive if $a \leq b, i . e . ~ a \wedge b=a$, and $b \leq c$ implies $a \leftrightarrow c$, in which'case asc. Logical structures associated, with trañsitive partial Boolean algebras satisfy the further condition:.

(v) If $a s_{i} b$ and $b s_{j} c$, there is an $L_{k}$ such that $a, b, c \varepsilon L_{k}$. 
Orthomodular posets are perhaps more familiar in the present cantext. They are structures' $P=\langle P, \leq, \vee, \wedge, 1,0, I\rangle$ where $\leq$ is a partial order on $\mathrm{P}$ and $\perp$ is an orthocomplementation; ' 1 and 0 arë greatest and least. elements, and $\vee, \wedge$ are the l.u.b. and.g.l.b. with respect to $s . P$ is weakly modular: avb exists whenever a is orthogonal to b (i.e. $\left.a \leq b^{\perp}\right)$, and if $a \leq b$, then $a v\left(b \wedge a^{\perp}\right)=b$. If any orthomodular poset it is possible to define a relation $C$ of compatibility: a $C$ b if there exist mutually orthogonal elements $a_{1}, b_{1}, \dot{c}$. such, that $a=a_{1} v c$ and $b=b_{1} v c$. (I.e., $a$ and $b$ are compatible if they are orthogonal except. for an overlap.) The representation theory for orthomudular posets has been established by Finch [3] (Theorems 1.1. and 3.1). The logical structures considered by Finch differ from those . associated with transitive partial Boolean algebras with respect to condition (iv). In [3] this is replaced by the weaker

(iv') Suppose $a s_{i} b^{+}{ }^{+}$for some $a, b \varepsilon L_{i}$. If $a \leq_{j} c^{\perp}$ and

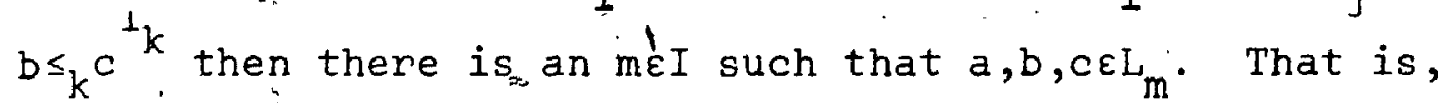
in an orthomodular poset we may have that $a, b \varepsilon L_{i}$; $a$, $c \varepsilon L_{k}$; and $b, c \in L_{k}$, but there is no $L_{m}$ containing $a, b, c$.

A compatible orthomodular poset is one which satisfies* the condition: ( $a \vee b) c c$ whenever $a, b, c$ are pairwise compatible: This is a necessary and sufficient condition " for every set of mutually, compatible elements to be contained in a Boolean subalgebra of P.

There is a very simple connection between orthotmodular 
posets and partial Boolean algebras: Every compatibleorthomodular poset is a transitive partial Boolean algebra and conversely.

I have presented the connection between orthomodular posets and partial Boolean algebras in terms of their representation theory. A direct proof of these remarks has been given by Gudder in [6]. His formulation is based.on the notion of an associative partial Boolean algebra: Suppose $a \leftrightarrow b$ and $b \leftrightarrow c, a, b, c \varepsilon L$. Then $L$ is associative, if $(a \wedge b) \leftrightarrow c$ iff $a \leftrightarrow(b \wedge c)$, and hence $(a \wedge b) \wedge c=a \wedge(b \wedge c)$. (By a lemma of Gudder and Schelp ([7] Lemm (3.3) a partial Boolean algebra is associative if and only if it is transitive.) Gudder shows that every associative partial Boolean algebra is a compatible orthomodular poset, and conversely: (Theorems 2.3 and 2.4. )

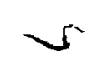

The partial Boolean algebra $\mathrm{B}(\mathrm{H})$ of closed linear subspaces of a separable Hilbert space is a transitrve partial Boolean algebra. The partial ordering is given by the subspace relation, and the operations of meet, join-and orthocomplement are representéd by the intersection, spari

- and orthogonal complement of subspaces. The zero of $B(H)$ is the 0 -dimensional subspace, and the unit is the whole opace $H$. The definition of $\leftrightarrow$ is $a \leftrightarrow b$, if $a C b$, i.e. if there are mutually orthogonal subspace $a_{l}, b_{I}, c$ such, that $b=. b_{1} v_{c}$, and $a=a_{1} v_{c}$. Equivalently; the subspaces of $\mathrm{H}$ form a compatible orthomodular poset. 
- To sum up: The representation of $\mathrm{H}$ as an orthomodular 'poset. is based on ifs ordex strueture since it is the fact that $H$ is partially ordered which is retained in the general concept of an orthomodular posel. The concept of a partial. Boolean algebra is based on the dompatibility structure of $\mathrm{H}$ since it preserves the fact that every triple of pairwise compatible subspaces is contained in a Boolean subalgebra of - H. 
3. Applications to the Problem of Hidden Variables.

Partial Boolean algebras were introduced by Kochen and specker in connection with the problem of hidden. variables. This is characterized as the problem of inbedding the non-commutative partial algebra of self-ądjoint operators ion $H$ into the commutative algebra of real valued functions on a classical probability-space. ,Their first theorem shows that there are no two-valued homomorphisms on a finite subalgebra of the partial Boolean algebra $B\left(E^{3}\right)$ of lines through a point in opdinary three dimensional, Euclidian space. It followis from this that thepe are no two-valued homomorphisms or $B(H)$. ( $I t$ is necessary to assume that the dimension of $\mathrm{H}$ be at least three.) Hence, by Theorem 0 there is no imbedding of $B(H)$ into a Boolean algebra. But the partial Boolean algebra of subspaces of $\therefore$ $\mathrm{H}$ is isomorphic to the subalgebra of idempotent operators on $\mathrm{H}$. Hence the partial algebra of physical magnitudes is not imbeddable into a commutative algebra.

By the equivalence of two-valued homomorphisms and . turvalued probability measures (or dispersion free statés), the absence of two-valued homomorphisms is an immediate corrollary toleason"s theorem. An independent proof of thịs corrollary wăs fïst,given by Bell. (See [1] p. 6.9f, for an expbsition of the proof.) Kochen and Specker's proof differs from Bell's since it does not depend on the denseness of the unit sphere in $\mathrm{H}$.

There is an interesting reformulation "of the imbedding 
problem in terms of the concept of a dogical structure (suggested by a paper of Maczynski [10]). This actualiy characterizes the problem of finding a weak imbedding of a partial Boolean algebra into a Boolean algebrax. The problem mag be further generalized by the concept of a homomorphic relation, but we shall not consider this here (see' [j] Sect. .

5). The interest of the reformulation from our point of view is that it clarifies, an important distinction, viz. the distinction between a Boolean theory based on the idempotent magnitudes of quantum mechanics and a: Boolean representation. of the algebra of idempotent magnitudes.

tet $\underline{N}=\left\{B_{i}: \sum_{i} \varepsilon I\right\}$

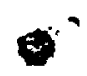
ciated with $\dot{B}(\mathrm{H})$ for a three dimetgional Hilbert space. $\left\{f j_{i}^{j}: B_{i}+B_{j}, i, j \varepsilon I\right\}$ is the set of inclusion bomomorphisms ${ }_{i}$ from $B_{i}$ into $B_{j} \cdot{ }^{i}=$ the identity map. Notice that " $f_{j}^{k} \circ f_{i}^{j}=f_{i}^{k}$, since $N$ is. a logicalstructure. Write $B_{i} \subseteq B_{j}$ if there is an $f$. A Boolear representation of $N$ is

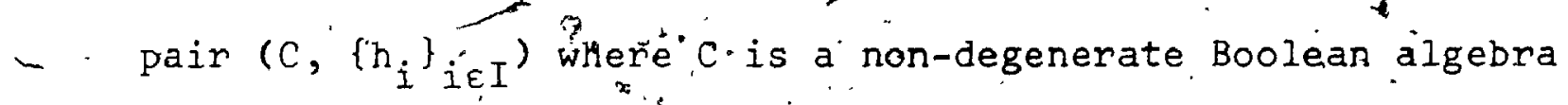
and each $h_{i}: B_{i} \rightarrow C$ is an imbedding carrying $B_{i}$ into $C$ such

* "that the following conditions are.satisfied:
(i) ${ }_{i \in I} \mathrm{Wh}_{i}\left[\mathrm{~B}_{i}\right]$ geperates $\mathrm{C}$.

(ii) If $B_{i}: \subseteq B_{i}, j$ then $h_{i}=h_{j} \mid f_{i}^{j}\left[B_{i}\right]$.

* (iii) Given âny ( $\left.C^{\prime},\left\{h_{i}^{\prime}\right\}_{i \in I}\right)$ șatisfying. (i) and (ii) there is unique homomonphism $h: C \rightarrow C$ ' such that $\mathrm{h}^{\circ} \mathrm{h}_{\mathrm{i} \cdot}=\mathrm{h}_{i}^{\prime}$.

The presence of "conatü (ii) smplies" that a Boolean represeptation of $N$ is a weak imbedding of $B(H)$ into $C$. . 


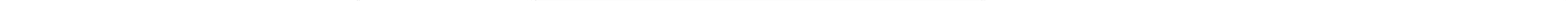


Suppose that ( $\left.\underline{F},\left(k_{i}\right\}_{i \varepsilon I}\right)$ is the direct product of - the $B_{i}$. That is to say, take the cartesian product $x=\pi_{i \varepsilon I-i} x_{i}$ of the spaces $X_{i}$. Associate each $Y \varepsilon \underline{F}\left(\ddot{X}_{i}\right)$ with a subset of $X$ by the mapping

$$
Y \rightarrow\left\{X \in X \mid x_{i} \in Y\right\}:
$$

'The field product $\underline{F}(X)$ of the $\underline{F}\left(X_{i}\right)$ is the field generated by the union of the images of the $\underline{F}\left(X_{i}\right)$ under this correspondence. $\underline{F}(X)$ contains an isomorphic copy of each $\underline{F}\left(X_{i}\right)$, and hence, an isomorphic image of each $B_{i} ;$

Condition ( $i i)$ distinguishes a Boolgan representation of the $B_{i}$ from their direct product. To see this consider an element $a$ in $B_{i} \leq B_{j}$. a is mapped by $k_{i}$ orfto the set of points in X whose $i=$ th coordinate is an ultrafilter in $B_{i}^{*}$ containing $a$. The image of $a=f_{i}^{j}(a)$ under $k_{j}$ is the set of points whose $j-t h$ coordinate is an ultrafilter in $B_{j}$ containing a. It is clear that in general $k_{i}(a) \neq k_{j}(a)$. Given the direct product, we may always obtain a. structure satisfying condition ( $i i)$, but this is not necessarily.a Boolean representation, 'To do this we proceed as follows. Let " $I$ be the ideal in $\underline{F}$ generated by ail elements of the form: $k_{i}(a)-k_{j}\left(f_{i}^{j}(a)\right) \cup k_{j}\left(f_{i}^{j}(a)\right)-k_{i}(a)$. For $X$; $Y$ in $\underline{F}$ define $X \sim Y$ if $X-Y \cup Y-X^{\prime} \in I, \underline{F} / I$ is the set of $\cdots$ equivalence classes of elements of $F$ under the relation $\sim$. Let $\phi: \underline{E}+\underline{F} / I$ be the canonical homomorphism from $\underline{F}$ into the quotient algebra $\underline{F} / I$. Write $k_{i}^{\prime}=\phi \circ k_{i}$. Then $(\underline{F} / I$, $\left.\left\{k_{i}^{\prime}\right\}_{i \varepsilon I}\right)$ is the direct limit of $N$. The direct limit is a Boolean representation if, and.only if, it is non-degenerate. 
It is always possible to construct a Boolean theory

based on the idempotent magnitudes in the $B_{i}$ simply by taking the direct product of the logical structure. This corresponds to Kochen and'Specker's trivial hidden variable construction. Such a theory is excluded by condition (ii), - for this condition makes the problem an imbedding problem. Rejecting this characterization of the problem is therefore equivalent to weakening the notion of a Boolean representation of a logical structure. But this overlooks the fact that exactly the same condition occurs in classical mechanics since, of the two representations, $\left.\left(\underline{F},\left\{k_{i}\right\}\right\}_{i \varepsilon I}\right)$ and $(F / I$, $\left.\left\{k_{i}^{\prime}\right\}_{i \in I}\right)$, classical mechanics uses the direct limit, not the direct product. Hence, coñdition (ii), or equivalently, the condition of weak imbeddability, can hardly be regarded as an ad hoc restriction, arbitrarily introduced to excilude classical extensions of the quantum theory.

Orthomodular posets are mainly associated with the "quantum logic" formulation of quantum mechanics. This represents a new axiomatic approach to the theory which. aims at generalizing von Neumann's presentation in terms of $>$ Hilbert space. The principal question here is: "To what extent can von Neumann's formulation be recovered without explicitly using the concept of Hilbert Space". This is a a mathematical investigation, motivated by mainly mathematical considerations. In this latter respect, it differs, not only. from Kochen and Specker's investigations, but from von Neumann's as well. 
Before von Neumann's treatise, the relationship

between wave mechanics and matrimmechanics was obscure.

For example, Dirac and Jordan viewed the similarity between

the two theories in terms of a "correspondence" between

the "ints" over which matrices in matrix mechanics and differential operators in wave mechanics are defined -- an idea which could not be consistently maintained as von Neumann showed. Von Neumann's formalization of the two theories in terms of Hilbert space was based on the observation that the algebraic structure of physical magnitudes is the same in both matrix and wave mechanics. This structure is represented by the non-commutative algebra of self-adjoint" operators on. a separable Hilbert space. The Hilbert space formalization is thus motivated by a question concerning the relationship between these two theories, and von Neumann presented the definitive clarification of the precise respect in which wave mechanics and matrix mechanics are equivalent.

In the quantum logic approach, the problem of hidden variables: consists in showing that in any acceptable generalization of the theory there are no dispersion free states:, Now the difficulties with the notion of an acceptable generalization/are obvious enough. However equally serious problems arise in connection with the notion of a generalization of quantum mechanics. 'For examplè, why should a generalization in any way preserve the algebraic structure of the theory? For this may be an inessential feature of the theory's formulation, and therefore, not properly part 
of any generalization of the theory. This is the criticism usually urged against hidden variable theorems:

These and similar difficulties are undercut by Kochen and Specker's formulation of the problem as an imbed-. ding problem. First, this restricts the issue to the relationship bétween two given theories, viz., quantum mechanics. and classical mechanics. The question is basically this: Is quantum mechanics a complete statistical theory? on is it statistical in the sense of classical statistical mechanics?In this case the absence of dispersion free states is the result of incomplete knowledge. Secondly, it completes the. program initiated by von Neumann: In Mathematical Foundations it was shown that the peculiarity of wave mechanics and matrix mechanics consists in the algebraic structure of their physical magnitudes. The solution to the imbedding problem shows, that this algebraic structure occurs essentially, and therefore cannot be regarded as a property of the theory's formulation.

0 
4. Idempotents as Prôositions.

To begin with, let us consider the relationship - betweenthe properties of a physical system and quanticties. $\therefore$ taking yalues in $\mathrm{R}$. Let $\Omega$ be'a subset of $\mathrm{n}$-dimensional

- Euclidian space. In classical particle mechaniçs a physical quaritity is represented by a function in $\mathrm{R}^{\Omega}$. Take a quantity $A$ and real nümber. $\lambda$ in the range of $f_{A} \varepsilon R^{\Omega}$. Then $f_{A}^{\prime}=\lambda$ (this is read "the value of $f_{A}$ is $\lambda$ ") represents a property of the physical. system S. More generaliy, given $A$ and a subset $U$ of $R, \dot{f}_{A} \varepsilon U$ (the value of $f_{A}$. lies in $U$ ) represents a property of $S$. .

- To define, the holding of a property we shall require the notion of a state of $S$. This must not be confused with a statistical state. A state is simply an event in the history of $S$. In classical mechanics an event is represented by a point $\omega$ in $\Omega$. As is well known, an event represented by $\omega$ is associated with a pure statistical state: the twovalued measure on $F(\Omega)$ determined by. $\omega$; but the ooncept of an event is not.a statistical concept.

The property $f_{A} \varepsilon U$ holds for 'S th and only if $S$ is in a- state such that $f_{A}(w) \varepsilon U$. Notice, in, von Neumann's terminology ([13] p. 249) f $f_{A} \in$ is then said to beewa property of the state $w$. This is misleading, as will be shown in connection with quantum mechanics.

Let is now consider the representation of properties of $S$ by idempotent magnitudes. Let $\Gamma$ denote the subset $f_{A}^{-1}(U)$ of $\Omega$. It is clear that $S$ has the property $f_{A} \cdot \varepsilon$ if and only 
if the state $\omega$-of-s lies in $r$. The property $f_{A} \varepsilon U$ is said to be associated with $\Gamma$. In general there are many properties $f_{A_{i}} \varepsilon U_{j}$ such that $f_{A_{i}}^{-I}\left(U_{j}\right)=I$ for some $U_{j} \subseteq R$ : Now let $x_{\Gamma}$ be the characteristic function of $\Gamma$, and let P'be a property associated with $\Gamma$. By the correspondence between properties of $S_{\infty}$ and subsets of $\Omega$, it follows that every property $\mathrm{P}$ is represented by the characteristic function $x_{\Gamma}$, in the sense that $P$ holds if and onlyl if $S$ is in a state $w$ such that $x_{\Gamma}(\omega)=1$. Since $x_{\Gamma}$ is two valued this is equivalent to $x_{\Gamma}(\omega) \neq 0$. This is the formulation employed by von Neumann ([13] Ch. III. 5).

Now the characteristic functions of the subseits of $\Omega$ are just the subset of idempotent elements in $R^{\Omega}$. The idempotents form $F$ Boolean ring with unit $I=x_{\Omega}$, i.e. a '. Boolean algebra. To simplify the notation, let $B$ denote the Boolean subalgebra of idempotents in $R^{\Omega}$. Elements of $B$ will be derioted by $a, b, c \ldots$. Wheñever lattice operations are used, it is assumed that they have been defined in terms of the ring operatidis in the usual way.

To summarize, we have seen that every magnitude may be replaced by a set of properties, and that every property corresponds to a two valued quantity, i.e. idempotent magnitude in $B$. The Comrespondence is not one-to-one, since very many properties are ąssociated with the same subset of $\Omega$, and therefore represented by' the same idempotent magnitúde. Since a is also a magritude in $\mathrm{R}^{\Omega}, \mathrm{a}=1$, (or $\mathrm{a} \neq 0$ ) is also a -property of $S$ just as $f_{A}=\lambda$ is a property of $S$. Here 
the property $a=1$, represty tis a whole class of properties of the forn $f_{A} E . U$.

That idempotents may be regarded as propositions is justified by the following considerations. For each a in, B we may define: a is truer $(1 . e . a=1, a \neq .0)$ if and only $\because$, if $\mathrm{S}$ is in a state $w$ such that $\mathrm{a}(\omega) \doteq 1$. (Since a is two valued, this is equivalent to $a(\omega) . \neq 0$.$) I.e. a is true$ if and only if $S$, has any (and therefore, all) of the'properties represențed by a.: Also,

$$
\begin{aligned}
& a^{\prime} \text { is true iff } 1-a(\omega)=1 \\
& a v b \text { is true iff } a(\omega)+b(\omega)-a(\omega) b(\omega)=1 \text {, }
\end{aligned}
$$

so that the lattice operations $'$ and $v$ in $B$ reppesent the operations of negation and disjunction of classieal logic. In a similar way the other propositional connectives may - be identified with the corresponding Boolean operations. [Note, strictly speaking $B$ should be regarded as the

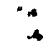

Lindenbaum-Tarski algebra of a suitable formal language $\underline{L}$, and truth then defined for sentences"for $\underline{L}$. For our purposes, this would introduce an unnefessary complication.]

The distinction drawn between states and statistical statés is expressed notationany as follows. Let a $\varepsilon$ B. If I write $\omega(a)$, this denotes the probability of the proposition a in the pure state determined by $\omega^{\prime}$ " But a(w) -denotes the trutivalue of a for the fitate $\omega$. . In other words, if I write w(a), $i . e_{a}$ if, $w$ appears as a function; then it denotes a probability measures byt when $w$ appears as an argument, it denotes a staté. 
Notice, in classical mechanigs we always have $\omega(a)=a(\omega)$.

This depends p the fact that the pure states; like the propositions are two valued. For this rason it is possible to define the truth of a proposition $a$ in $B$ by writing

$a$ is true-iff $S$ is in a state ' $w$ 'such that $w(a) .=" 1$.

$a^{\prime}$ is true iff $1-w(a)=1$.

$a \vee b$ is true iff $\omega(a)+\omega(b)-\omega(a) \omega(b)=1$.

This definition is formally equivalent to the one given earlier. "But the conception of truth which underlies the tพ้ deffinitions is very different. This definition identifies truth with probability equal to 1 , so that a proposi-) tion is true only if the statistical state of $S$ assigns it probability 1. That is to say, for a proposition a to be true, the system must be in a pure statistical state $\omega$ such that $\omega(a)=1$.

In classical mechanics each $\omega$ in $\Omega$ determines a twovalued homomorphism $h: B \rightarrow Z_{2}$. Each $h$ corresponds biuniquely to a two-valued measure $w$ on $F(\Omega)$. Substituting $h$ for the probability measure determined by wields a formally equivalent definition of truth. This definition is related to the one given in vam, Fraassen [12] as follows. A possible world is a classical truth value assignment to the propositions of B. Each such world is represented by a two-valued homomorphism. A proposition is true only if it is true in some possible world, i.e. only if $h(a)=1$, for some $\mathrm{h}: \mathrm{B} \rightarrow \mathrm{Z}_{2}$. This is a necessary condition for the truth ofd. (a is true if this truth value assignment is deter- 
mined by the actual world.) The mistake underlying the identification of truth with probability equal to 1 is transparent. It is, less obvious that exactly the same misconception underlies this definition.

First, notice that if a possible world is represented by a state or event (rather than a two-valued homomorphism), the resulting definition coincides with the one given here, i.e. with $a(w)=1$. It is not the concept of a possible world which poses a problem, but the interpretation of a possibile world as a two-valued.homomorphism. For this has the consequence that the truth of an atomic proposition is defined only if the truth or falsity of every other proposition als also specified. But this is certainly ' not required by the correspondence theory of truth. To put 1 the truth of the proposition (a) "It is raining" depends, net on the truth or falsity of every other proposition, but justy on the state of the weather. This is independent of whether yer not our knowledge of the truth of (a) depends on our. knowledge of the truth of other propositions. It is this simple insight which is preserved in the correspondence. theory, and which is given up when, in the definition of the truth of (a), it is required that $h(a)=1$.

We may summarize this discussion of the definition of "truth" in classical mechanics. Classical'y a state is associated with a two-valued measure and a two-valued homomorphism. Because the Boolean algebra of subsets of $\Omega$ 
is perfect and reduced, this association is biunique. There are therefore three formally equivalent definitions of "a is true": A) $a(w)=1, B) \omega(a)=1$ and $C) h(a)=1$. In the first definition the truth of a is exclusively determined by the state of $S$ : A proposition a is true if and only if the system has (any) one of the properties represented by a. Each of the other two definitions imposes an additional requirement on the truth of $a$. In the case of $B$; a must have a probability equal to 1 . If the definition is $h(a)$ $=1$, then the truth of a requires that the truth or falsity of every other proposition in $B$ is also given. The formal equivalence, which holds classically, does not mean that $A$, $B$, and $C$ are explications of equivalent conceptions of truth. The conception underlying $B$ is remarkably like the pragmatic theory of truth, while $\dot{c}$ is similar to the doctrine of internal relations of the coherence theory. Both conceptions of truth confuse the meaning or reference of the pro- I position "a is true" with considerations that are strictly evidential in character.

Thus far we have concentrated on classical mechanics, but the situation in quantum mechanics is exactly analogous. The idempotent magnitudes, i.e. the propositions $a$ in the partial Boolean algebra $N$ are $P$ jection operators acting on a suitable Hilbert space $H$. A state or event of a system S. is represented by a (unit) ray $K$ in H. Each event $K$ is assciated with a pure statistical state. In quantum mechanics, statistical states are given by measures on the 
closed linear subspaces of $\mathrm{H}$. The pure state associated - with $k$ is determined by taking the square of the norm of the projection of a unit vector lying in $k$ onto each subspace of $\mathrm{H}$. Since by Theorem 12 of von Neumann [13], there is a one-to-one correspondence which associates each projection operator with the subspace in $\mathrm{H}$ which is its range, this deternines a probability measure on N. Recall, in classical mechanics a statistical state is a measure on the field $F(\Omega)$ of subsets of $\Omega$. There the correspondence between subsets and propositions is trivial. Noté, atoms in $B$ are the characteristic functions associated with singleton subsets $\{\dot{\omega}\}$ of $F(\Omega)$. N is also atomic. An atom in $N$ is a projection operator onto a one-dimensional subspace of H. Thus in each theory there is a one-to-one correspondence. between events and atomic propositions.

Quantum mechanics is probabilistic in the sense that the set $S$ of statistical states does not include two-valued measures. This means, that the expectation (or average). value is never dispersion free for all magnitudes -- even in the case of pure statistical states. But exactly the same rmagnitudes, and therefore, exactly the same propositions, occur in both classical and quantum mechanics; that is, the propositions of both $B$ and $N$ make assertions about properties of. physical systems, not ensembles of such systems. In quantum mechanics there are no statistical states which determine a probability of 1 or 0 for every a in $N$, and which may therefore be regarded as two-valued truth value 
assignments to the propositions of $\mathrm{N}$. Although the statistical state determines the probability of every a in $N$, the corresponding event does not determine the truth values of all propositions. .

Suppose that the event represented by $k$ determines the truth or falsity of a. Then the situation is exactly as described for classical mechanics, and the truth of $a$ ' and $\mathrm{a} v \mathrm{~b}$ is defined by writing

$$
\begin{aligned}
& a^{\prime} \text { is true iff } 1-a^{\prime}(K)=1 \\
& a \vee b \text { is true iff } a(K)+b(K)-a(K) b(K)=1 .
\end{aligned}
$$

That is, the partial operations ', $V$ of $N$ represent the logical operations of negation and disjunction of classical. logic.' (Recall.that in a partial loolear algebra a $v$ b exists iff $a$ and $b$ are compatible.)

- of course, the identity

$$
a(K)=K(a)
$$

cannot hold in quantum mechanics. It is possiblè to make. it hold by identifying the truth value of a in the state $k$ with the probability assigned to a by the statistical state determined by $K, i . e$. by stipulating that $a(K)^{\circ}=K(a)$. But, there is no more justification for identifying truth with probability in quantum mechanics, than there is in classical mechanics.

It follows from what has betn sajd so far that an. event does not determine all properties df s. Here it important to be extremely clear. Let us consider a specific property P. Then there are states $K$ such that $K$ does not determine whether $P$ hold or fails to hold. But $P$ is a 
property of $S$ and it' is always determinate whether or not $P$ holds for $S:$ The answer to this question is contained in $S$, though not in every event. in the history of $S$. The . determinateness of the holding of $\mathrm{P}$ is completely independent of whethenpor not the holding of $P_{\text {, }}$ is determined by every state of $S$. I regard this claim as central to the logical interpretation of quahtum mechanics.

This is obscure if it is assumed that $P$, is a property. of a state rather than a property of $S$ and that $S$ has only. a single state $K$. . It is true that there is a single statistical state which describes $S$. The uniqueness of the statistical state of $\dot{S}$ is requiréd by quantum mechanics;" as it is by classical mechanics. The claim that $S$ has a unique statistical state must not be confused with a certain. interpretation of mixed states. If $S$ is represented.by the mixed state

$$
W=\sum_{i} w_{i} K_{i}, \quad \text { is }
$$

the this is understood to mean that $S$ is in the pure state $K_{i}$ wth weight $w_{i} \cdot$ But we may also have $W=\sum_{i} w ! K !$

with $w_{i} \neq w_{i}^{r}$ and $k_{i} \neq k_{i}^{\prime}$. That is, there is no theoretical basis for singling, out any one representation of $W$. But of course this is completęly independent of whether or not there is any basis for supposing $S$ to be in a unique 'statistical state. The point is 'sjmply this: There is a unique representation of the statistical state of $s$ in the theory, bu't there is no unique repressentation of the mixture $W$ in terms of pưre states. 


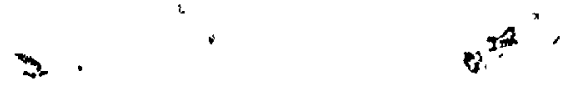

In guantum mechanics a system $S^{\prime \prime h a s ~ a: ~ s i n g l e ~ s t a t i s-~}$ - o tical state. But S has many states -- enough to determine the truth value of each a in N. In both classical mechanics: andimuantum mechanics, every $a$ in or $B$ is true or false, and ' $j f$ a is false, than $a$ ' is true. This is a consequence of the fact that both algebras are idempotent, hence every $a$ in $N$ or $B$ takes orily the values $O$ (false) and $I^{\text {(true). }}$ But $B$ is also semi-simple, and therefore admits two-valued measures. The correspondence between two-valued measures 0 and events means that each event, determines a two-valued truth value assignment to all the propositions of $B_{i}$. This is to be contrasted withol which is not semi-simple (Theorem $I$ of $[9])^{\circ}$, hence there is no extension of $\mathrm{N}^{\circ}$ which recovers the comrespondence between everts and otwo-valued measures. The structure of $\mathrm{N}$ makes it necessary to distinguish an event of riom a possible woṛld since intuitiyely, a possibile world should determine aíl properties of $S$. But $S$ has many more properties than are represented by the propositions whoseatruth is determined by $k$. Hence $k$ 'cannot be regarded as a possible world in this sense. The sitution is rather as follows:- In clăssical mechanics a single point"w in $\Omega$ represent a possible world, since a* single event determines all properties of $S$. In quantum Ifechanics, a possible world is represented only by the whole loxical space $N$, since no one event determines all properties of $\mathrm{s}$. This suggests that the usefulness of the concept of a possible "world, is restricted to the classical pase. 
In quantum mechanics a pure statistical state of $S$ is significantly probabilistic in the sense that pure states are not degenerate statistical states as in classical mechanics. But unlike classical statistical mechanics, this does not arise from an incompleteness in the theory, since $i t^{\circ}$ is imposisible to introduce two-valued measures." given the logical structure of $N$. In classical statistical mechanics the degenerate statistical states, i.e. the twovalued mewures, are recoverable by imbedding the algebra of idempotent macroscopic magnitudes into an atomic Boolean algebra. But $\mathrm{N}$ is atomic, and there are no (proper) extensions of $\mathrm{N}$; in particular there are no Boolean extensions. So far it has been shown that the classical concepts of truth and logical structure are largely independent, and that the concept of truth is the same in both classical and quantum mechanics. In particular, any formal language $\underline{L}$, which is based on $N$, i.e. for which $N$ is the LindenbaumTarski Algebra, is bivalent. L differs from a classical

() language in the following respect. A sentence $\phi$ of $\underline{L}$ corresponds to an element a of the logical structure $N$. This is exactly as in classical logic. But unlike the classical case, a is never associated with a two-valued homomorphism on $\mathrm{N}$. To put this slightly differently: A Boolean representation of the fact that every sentence $\phi$ is true or false requires that the corresponding $a$ in $B$ is representable by the sequerice $k(a)$ of its truth values under. all possible truth value assignments. Semi-simplicity in- 
sures that this is always the case. Now every sentence $\phi$ of $\underline{L}$ is true or false, and only true or false. For $\underline{L}$ is bivalent if $\mathrm{N}$ is idempotent, i.e. if for every a in $\mathrm{N}$, a^a $=a$. But the failure of semi-simplicity means that. there is no Boolean representation fhis fact. Whether every proposition a in $N$ (and hence, every sentence $\phi$ in is true or false depends exclusively on $a$. The bivalence of $\underline{L}$ is independent of how the corresponding propositions in $\mathrm{N}$ are interrelated. This is obviously not true if bivalence is represented by semi-simplicity.

'It' has been maintained (e.g. by van Fratssen: [12] and Friedmann \& Glymour [4]) that there is a problem with applying the classical concept of truth to elements of $\mathrm{N}$. This problem does not arise for propositions in B or in a maximal Boolean subalgebra $B_{i}$ of $N$, since $B$ and $B_{i}$ each. admit two-valued homomorphisms. If the discussion of this section is correct, semi-simplicity is irrelevant to the classical concept of truth, so that new "semantic analyses". of quantum mechanics are completely ancillary. 
5. "Anomolies".

I shall now briefly consider the bearing of this discussion on the paradoxes or anomolies of quantum mechanics. (A complete discussion is contained in a forthcoming paper with Jeffrey Bub.) : It seems likely that all of the paradoxes have essentially the following form. There exist two statistical states, one pure, the other a mixture, which are the same for a given class of idempotents, but which are generally distinct. The pure state correctly characterizes the system (i.e. it is confimed experimentally). Such a state generates a collection of propositions asserting the probability that the system has a certain property. A mixed sitate gives the probability that the system is in a given,pure state, and from this the probability that $S$ has a certain property is inferred. For example suppose we are interested in a property $R$ but that the pure state of $S$ is neither 0 nor' 1 for the proposition a in $\mathrm{N}$ representing $\mathrm{P}$. One might suppose that $S$ is really characterized by a mixture, so that $S$ is in a pure state $K$ with a certain weight $w$ such that $K$ assigns $a$ a probability of 1 , on 0 . But this is exoluded thepretically (by the fact that pure states are not reducible to mixtures) às well as experimentally.

- The situation just described arises in the two slit experiment. '(This is the only case I shall explicitly consider.) Recall that the statistical state of the electron is experimentally determined by examining the diffraction pattern which appears on the emulsion opposite the screen aften verv manv electrons have' been emitted by the source. 
The electrons are emitted one at a time and at intervals of any length. The statistical state $K$ associated with both slits open is not the weighted sum of the pure-statistical states $k_{\alpha_{i}}$ which assign a probability of 1 to the propositions $a_{i}:$ " "The electron passes through slit $i$ " $(i=1,2)$. The pure state exhibts interference, while the mixed state does not. Now the fact that the statistical state is not the mixture but another pure state is not paradoxical. By itself this is no more puzzling than the relativistic replacement of the classical addition theorem for relative velocities. It is clear that an anomoly arises. only if there is some reason to suppose that the statistical state must be represented by a mixture of the $K_{\alpha_{i}}$.

The basic idea seems to be that if the probability of $a_{i}$ is neither 1 nor 0 in the state $k$, then the electron is in some sense indeterminate with respect to the property $a_{i}=1$ (or $a_{i} \neq 0$ ). This means that neither $a_{1}$ nor $a_{2}$ is true or. false. On this basis it is often suggested that quantum mechanics requires some thorough-going revision in our space-time concepts: the twc slit experiment is anomolous given our classical and relativistic conceptions of spącetime. But how seriously this suggestion should be considered is unclear since it has never been seriously developed: I In any case it overlooks the fact that quantum fechanics, like classical mechanics and relativity, assumes that the symmetry group pf all physical systems is a subgroup of the manifold mapping group, and that therefore, the theory makes pre- 
cisely the same continuity and differentiability assumptions as the se theories. Hence on this suggestion, quantum mechanic' is a fundamentally incoherent theory, But the theoretical and experimental success of quantum mechanics is simply' too great for this conclusion to be seriously: considered.

-That the whole problem is misconceived, is immediate from the analysis of this paper: The statistical state of thelectron is indeed the pure state. This state exhlbits interference, and is not to be replaced by a mixture. The pure state like all of the statistical states of the theory, is significantly probabilistic: i.e. it assigns a probability which is not dispersion free on very many propositions in. $N$. In papticular, it is not 0 or $I$ for $a_{i}$. But each $a_{i}$, is true or false. Moreover, this holds for every proposition in. N. A difficulty arises when one attempts to express this fact. by a simultaneous truth value assignment to all propositions in $\mathrm{N}$, since this is possible only if $\mathrm{N}$ is imbeddable into a Boolean algebra.

More generally, quantum mechanics is indeterministic in the sense that the pure statislical states of the theory are' not-degenerate measures concentrated on 0 and 1 as in classical mechanics; herce the maximal amount of information concerning a physical system is Agnificantly probabilistic. : This arises from the fact that certainproperties are independent, as are the idempotents hich represent them. More exactly, the properties are strongly independent in the sense 
- that they are related to other properties in a way which excludes their being related to each other. This is the significance of incompatibility.

Indeterminism iric this sense must not be confused with the very different concept of indeterminateness The theory is indeterministic "or significantly statistical in' the. sense that the pure states take values in the open interval $(0,9)$ : "The thesis that the theory is indeterminate holds that there are properties $P$ such that' $P$ neither holds nor fails to hold of $S$. This is not implied by indeterminism nor is it in any way required by the view that the theory provides a maximal amount of information concerning the system. Rather, indeterminateness is suggested by essentially two mistaken ideas. The first of these is that incompatible. -propositions are somehow inconsistent. But just. the opposite is the-case: since a pair of propositions are incompatible, they cannot be inconsistent.

The second mistake concerns the conception of the truth of atomic propositions. According to the correspondence theony of truth an atomie proposition a in $N$ is true if and only if the prqperties represented by a hold of $S$. This definition is independent of the semi-simplicity of $\mathrm{N}$, so that at any given instant exactly one proposition in each $B_{i} \subset N$ is true of $S$. The essential point is that the absence - of a simultaneours truth value assignment does not imply that the properties of $S$ which, on the arccount given here, are supposed to obtain, cannot obtain simultaneously. The 
opposite yiew rests ōn a simple equivqcation. A simultaneous truth value assignment is a two-valued homomorphism. That simultaneous truth value assignments do not exist is a fact about the structure of $\mathrm{N}$ which has nothing to do with what occurs simultaneously. The properties of $S$ which obtain simultaneously include incompatible properties. But their logical structure excludes the existence of two-valued homomorphistn , and hence, of a simultaneous truth value assignment to the corresponding idempotents. At any one

- instant the' system is characterized by exactly one class of - properties from each $B_{i} c N$, and all such classes of properties obtain at the same time. But there is no Boolean representation of this fact.

To sumfiarize this part of the discuseion: There are 'two different accounts of indeterminism which are historically important. The first, which apparentiy goes back to Aristotle, rejects bivalence: A theory is indeterministic if it assmes. that there are propositions whose truth value is indeterminate. The second, represented by the quantum theory, retains bivalence whife rejecting semi-simplicity. An indeterministic: theory is ther characterized by, the absenoe of two-valued, homomorphisms, and therefore, of two-valued measures. The coherence of indeterminateness seems to rest on the Aristotelian metaphysic of act and potency. But nothing of. this sort is required by the indeterminism of quantum mechanics. This form of indeterminism implies that there is no Boolean represeptation of the propertiesobtaining at a given time; 


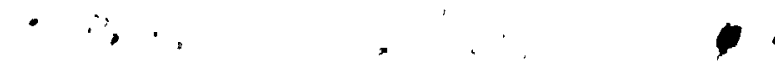

yêt for any property $P$ it is completely determinate whether or not $P$ holds.

The anomolous character of the two sit experiment' depends on the assumption that the system is indeterminate with respect to the property $a_{i}=1$, if the statistical state is $k \neq K_{\alpha_{i}}$. This assumes that the usual notions of tmuth and falsity pake sense only in the case of propositions which form a Boolean algebra: This view lies at the basis of both the Copenhagen and hidden variable interpretations of the quantum theory. According to the Copenhagen interpretation, at each instant the system "projects" exactly one maximal Boolean subalgebra of $\mathrm{N}$; in a hidden variable interpretation all Boolean sưbalgebras are represented but their structure is Boolean: The difference is that the Copenhagen interpretation is willing to consider systems with properties corresponding to at most one maximal Boolean subalgebra in the logical structure associated with N. Now the implicit restriction of $\left\{B_{i}: i, \varepsilon\right.$ I $\}$ to maximal Boplean. subalgebrass has an interesting consequence. If $B_{i}, B_{j}$ are maximal Boolean subalgebras, then $B_{i} \subseteq B_{j}$ implies $\dot{B}_{i}=B_{j}$, i.e: the logical structure $\left\{B_{i}: i \varepsilon M\right\}$ consisting of all maximal Boolean subalgebras is totalfy unordered by s. In this case condition (ii) of Section 3 is trivially satisfiéd, so that the direct Iimit of the $B_{i}$ coincides with the direct product, which is a Boolean representation of $\left\{B_{i}: i \varepsilon M\right\}$. Thus both the Copenhagen and hidden variable inter-. pretations are committed to/representations of $\left\{B_{i}: i \varepsilon M\right\}$ 
which are semi-simple: In the hidden rafiable interpretation this requires dropping condition (ii), while in the copeshagen interpretation this is accomplished by replacing $\left\{B_{i}: i \varepsilon I\right\}$ with $\left\{B_{i}: i \varepsilon M\right\}$. In effect both views fail to consider the possibility that the properties of physical systems exemplify the logical structure of $N$. I.e. both views completely fail as accounts of the significance of the transition from classical mechanias to quantum mechanics. Itt should be remarked that the hidden variable interpretation is at least coherent. The idea that a system projects a single $B_{i}$ at each instant has the absurd consequence that the system must somehow anticipate the deciaion of the experimenter to measure a magnitude associated with $B_{i}$. This consequence is avoidable only if measurements are. regarded as a theoretically opaque subclass of physical interactions. It is a strange comment on current investigations in foundations of physics that both possibilities are seriously considered and widely entertained.

[The possibility of a classical theory of the maximal magnitudes was noted by Wiener and Siegel ([14], Appendix); and independently, by Gudder [5], who proved this theorem in a more general context. The discussion here follows the . very, elegant "presentation of Maczynski.]

In conclusion, I wish to compare the discussion in Sèction 4. with von Neumann.[13] Ch. III. 5, "Projections às Propositions". Prima facie there.is only the slightest difference between the conception of truth present here and the one implicitly assumed by von Neumann. "But the, 
difference has importan't consequences. Adopting von Neumann's approach, one is led to propose an additional class of nonunitary time-transformations. This is the content of the projection postulate, which may be simply explained as follows: Let us assume that a system $S$ is in à pure state represented by a unit ray $K_{\psi}$ in $H$ (the Hilbert space associated with $S$ ). Now suppose we find that the value of a magnitude $A$ is $a_{i}$. The value $a_{i}$ is associated with a ray $k_{\alpha_{i}}$ in $H . \quad K_{\alpha_{i}}$ represents the pure statistical state which assigns the property $A=a_{i}$ a probability equal to one. The projection postulate requires that the statistical, state of $S$ undergo a transition: $k_{\psi}+K_{\alpha_{i}}$ which is generally discontinuous. Since the dynamics of the theory considers only continuous transitions, the projection postulate re-. presents an additional hypothesłs.

Essentially the same idea underlies the characterization of the probabilities of quantum mechanics as "transition" probabilities." The probability $\left|\left(\psi, \alpha_{i}\right)\right|^{2}$ which $k_{\psi}$ assigns to $A=a_{i}$ is not the probability that the property $A=\dot{a}_{i}$ obtains, but rather, the probability that it will obtain. Wis requires that $S$ undergo a transition from the state $k_{\psi}$ to $K_{\alpha_{i}}$. The problem of finding a theoretical account for such stochastic transitions has come to be known as the "measurement problem", since transitions of this type " are supposed to occur whenever a measurement is performed. The projection postulate is simply a precise characterization of this class of transitions. 
If the analysis of this paper is correct, the projection postulate results from a misconception of the logical structure of the theory: there is nothing about the logical structure which requires that a proposition is true only in ceptain statistical. states. The projection postulate is therefore quite clearly ancillary to an understanding of the theory. Similarly, since the measurement problem requires the occurence of. transitions of the kind described by the projection postulate, it follow that this 1 -cannot be a real difficulty for the theory. 
References

[1] 'J. Bub, The Interpretation of Quantum Methanics, Reidel, 1974.

[2] J. Bub and W. Demopoulos, "The Interpretation of

$\therefore$ Quantum Mechanics", Boston Studies in the Philosophy of Science Vol. KII, ed. qy R.S. Cohen and M.J. Wartofsky, Reidel,

[3] P. D. Finch, "On the Structure of Quantum Logic", Journal of Symbolic,Logic 34 (1969) 275-282..

[4] M. Friedman and C. Glymour, "If Quarita HAd Logic", Journal of Philosophical Logic 1 (1972) 16-28.

[5] S. Gudder, "On Hidden-Variable Theóries", Journal of Mathematical Physics 11 (1970) $431-436$.

[6], S. Gudder, "Partial Algebraic Structures Associated with Orthomodulaŕ Posets", Pacific Jounnal of Mathematics 41 (1972) 717-730.

[7] S. Gudder and R. Schelp, "Coordinatization of Orthocomplemented and Orthomodular Posets", Proceedings of the American Mathematical Society 25 (1970): 229-237.

[8] S. Kochen and E. P. Specker", "Logical, Structures Arising in Quantum Theory", The Theory of Models; 1963 Symposium at Berkeley, compiled by 3., Addison et.ak., North-Holland; 1965.

[9] S. Kochen and E. P. Specker, "The Probiem of Hidden Variables in Quantum Mechanics", Journal of Mathematics 


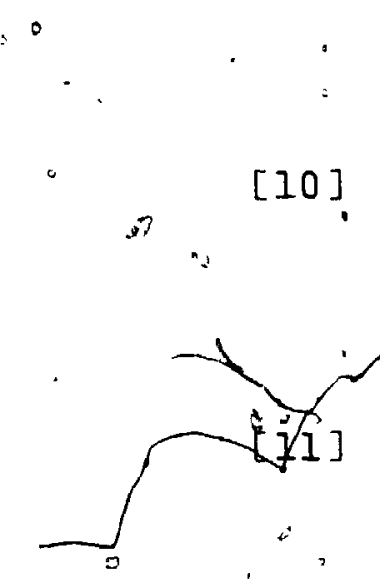

[10]. M. J. Mayzynski, "Boolean Properties of Observables in Axiomatic Quantum Mechgnics", Reports on Mathematical Physics 2 (197i) $135-150$.

H." Putnam, "Is Logic Empirical?", Boston Studies in. the Philosoply of Science, Vol, $\underline{V}$, ed. by R.S. Cohen and M.J. Wartofsky, Reidel, 1969 .

[12]: B. van Fraassen, "Semantic Analysis of Quantum Logic", Contemporary Research in the Foundations and Philosophy of Quantum. Theory, ed. by C.A. Hooker, Reidel, 1973.

[13] J. von Neumann, Mathematical "Foundations of Quentum Mechanics, Princeton University Press, 1955.

[14] N. Wiener and A. Siegel, "The Differential-Space Theory of Quantum Systems", Il Nuovo Cimento supplement to ${ }^{\circ}$. Vol II, series X $(7955) 982^{\circ}-1003$. 
Anderson, J.L. Prineiples of Relátivity Physics, Academic Press, 1967.

Birkhoff, G. and Von Neumann, $\mathrm{J}$. "The Logic of Quantum Mechanics", Annals of Mathematics 37 (1936) 823-843.

Born, M. The Born-Einstein Lètters: The Correspondence Between Albert Einstein and Max and Hedwig Born: 1916-1955, Walkér and Company, 1971.

Bub, J. (1) "On the Possibility of a Phase Space Reconstruction of the Quantum Statistics: A Refutation of the Bell-Wigner Locality Argument", Foundations of Physics 3 (1973) 29-44,

(2) "On the Completeness of Quantum Mecharics" in Contemporary Research in the Foundations and Philosophy - of Quantum Theory, ed.'by C. Hooker, Reidel, 1974. 1974. (3) The Interpretation of Quantum Mechanics, Reidel,

Bub; J. and Demopoulos, W. "The Interpretâtion of Quantum Mechanics", 'Boston Studies in the Philosophy of Science Vol. XIII, eqd. by R.S. Cohen and M.J. Wartofsky, Reidel 1974:

Einstein, A.(1) What is the. Theory of Relativity?", (1919)

Essays in Science, Philosophical Library, 1934. (2) "Quantum Mechanics and Reality" (1948), in The Born-Einstein Letters: The Correspondence Between Albert Einstein and Max and Hedwig Born: 1916-1955, Walker and Company, (1971) 16,8-173. (3) Autobiographical Notes (1949), Albert Einstein:

- Prilosopher-Scientist (ed. by P.A. Schilpp), -Harper, 1959 .

Fay, G. "Transitivity of Implication in Orthomodular Lattices", Acta Sci. Math.. (Szeged) 28 -(1967) 267-270.

Finch, P.D. "On the Structure of Quantum Logic", Journal of Symbolic Logic 34 (1965) 279-282.

Friedman; M. and 61ymour, C. "If Quanta Had Logic". Journal of Philosophical Logic I (1972) 16-28.

Gleason, A. "Measures on the Glosed Subspaces of Hilbert Space" ". Journal of Mathematics and Mechanics $\frac{6}{8}$ (1957)

Greechie, R. and Gudder, S. "Quantum Logics" in Contemporary Research in the Foundations and Philosophy of Quantum: Theory, ed. by C. Hooker, Reide1, 1974. 
Gudder, S: (1) "On Hidden-Variable Theories", Journal of Mathematical Physics 11 (1970) $43^{\circ} 7-436$.

(2) "Partial Algebraic Structures Associated with Orthomodular Posets", Pacific Journal of Mathematics 41 (1972) 717-730. Probability, Statistifal Inference and Statistical. Theories of Science, ed. by C.Hooker and W. Harper, Reidel (forthcoming).

Gudder. S. and Greechie R. "Is Quantum Logic a Logic?", Helv. Phys. Acta 44 (1971) 238-240.

Gudder S. and Schelp, R. "Coordinatization of Orthocomplemented and Orthomodular Posets", Proceedings of the American Mathematical Society 25 (1970) 229-237.

Halmos, P. Lectures on Booleah Algebras, Van Nostrand, 1963.

Jauch, J. Foundations of Quantum Mechanics, Addison-Wesley, 1968 .

Kochen S. and Specker, E.P. (1) "Logical Structures Arising in Quantum Theory", The Theory of Models, 1963 Symposium at Berkeley, compiled by J. Addison et.al. NorthHolland, 1965 .

(2) "The Problem of Hidden Variables in Quantum Mechanics", Journal of Mathematics and Mechanics 17 (1967) 59-87.

MacNeille, H.M. "Partially Ordered Sets", Trans Am. Math. Soc. 42 (19.37). 416-460.

Maczyniski, M.J. "Boolean Properties of Observables in Axiomatic Quantum Mechanics", Reports on Mathematical Physics.2 (1971) 135-150.

Peremans, พ. "Embedding of a Distributive Lattice into a Boolean Algebra", Nederl. Akad. Wetensch. Indag, Math. 19 (1957) 73-81.

Putnam, H. "Is Logic Empiricals", Boston Studies in the Philosophy of Sciencel, Vol. v, ed. By R.S. Cohen and M.J. Wartofsky, Reidel, 1969.

Rasiowa H. and Sikorski, R. The Mathematics of Metamathematies, P.W.N., 1963.

Sikorski, R. Boolean Algebras (2nd ed), Springer-Verlag; 1964. 
117

Trautman, A. (1) Lectures on General Relativity, Brandeis Summer Institute, 1964, Prentice Hall, 1965.

(2) The General Theory of Relativity, Nuclear Energy Information Center of the Polish Government, 1968 .

van Kampen, N.6. "Fundamental Problems in the Statistical $\because \therefore$ Mechanics of Irreversible Processes", Fundamental

- Problems in Statistical Mechanics, Proceedings of the NUFFIC International Lumen Course in Science at Nijenrode Castle, The Netherlands, 1961, compiled by E.G.D. Cohen, North-Holland, 1962.

van Fraassen, B. "Semantic Analysis' of Quantum Logic" in Contemporary Research in the Foundations and Philosophy of Quantum Theory, ed. by C. Hooker, Reidel, 1974.

van Newman, J. Mathematical Foundations of Quantum Mechanics, Princeton University Press, 1955.

Whey, H. Philosophy of Mathematics and Natural science (Rev. ed.), Princeton:University Press, 1949.

Wiener, N. and Siege, A. "The Differential-Space Theory of Quantum Systems", Il Nuovo Cimento, supplement to

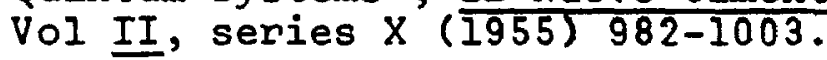

ข

- Zierler, N. and Schlesinger, M. "Boolean Embeddings of Ortho-Modular Sets and Quantum Logic", Duke Journal 32 (1965) 251-262:

$\therefore 0$

eg 